\title{
Compositionality for Markov Reward Chains with Fast and Silent Transitions
}

\author{
J. Markovski ${ }^{\mathrm{a}, *, 1}$, A. Sokolova ${ }^{\mathrm{b}, 2}$, N. Trčka ${ }^{\mathrm{a}}$, E.P. de Vink ${ }^{\mathrm{a}}$

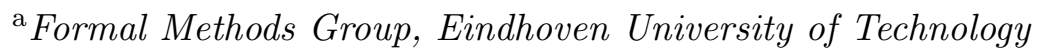 \\ Den Dolech 2, 5612AZ Eindhoven, The Netherlands \\ ${ }^{\mathrm{b}}$ Computational Systems Group, University of Salzburg \\ Jakob-Haringer-Straße 2, 5020 Salzburg, Austria
}

\begin{abstract}
A parallel composition is defined for Markov reward chains with stochastic discontinuity, and with fast and silent transitions. In this setting, compositionality with respect to the relevant aggregation preorders is established. For Markov reward chains with fast transitions the preorders are $\tau$-lumping and $\tau$-reduction. Discontinuous Markov reward chains are 'limits' of Markov reward chains with fast transitions, and have related notions of lumping and reduction. Markov reward chains with silent transitions are equivalence classes of Markov reward chains with fast transitions and come equipped with the lifted preorders $\tau_{\sim}$-lumping and $\tau_{\sim}$-reduction. In total, six compositionality results are presented. Additionally, the parallel operators involved are related by a continuity result.
\end{abstract}

Keywords: Markov reward chains, fast transitions, silent transitions, parallel composition, aggregation

\section{Introduction}

Compositionality is a central issue in the theory of concurrent processes. Discussing compositionality requires three ingredients: (1) a class of processes or

\footnotetext{
* Corresponding author.

Email addresses: j.markovski@tue.nl (J. Markovski), anas@cs.uni-salzburg. at (A. Sokolova), n.trcka@tue.nl (N. Trčka), evink@win.tue.nl (E.P. de Vink).

1 This research has been funded by the Dutch BSIK/BRICKS project AFM 3.2.

2 Supported by the Austrian Science Fund (FWF) project P18913 and by the EU ArtistDesign Network of Excellence on Embedded Systems Design.
} 
models; (2) an operation to compose processes; and (3) a notion of behaviour, usually given by a semantic preorder or equivalence relation on the class of processes. For the purpose of this paper, we will have semantic preorders and the parallel composition as operation. Therefore, the compositionality result can be stated as

$$
P_{1} \geqslant \bar{P}_{1}, P_{2} \geqslant \bar{P}_{2} \Longrightarrow P_{1}\left\|P_{2} \geqslant \bar{P}_{1}\right\| \bar{P}_{2}
$$

where $\mathrm{P}_{1}, \mathrm{P}_{2}, \overline{\mathrm{P}}_{1}$, and $\overline{\mathrm{P}}_{2}$ are arbitrary processes and $\|$ and $\geqslant$ denote their parallel composition and the semantic preorder relation, respectively. Hence, compositionality enables the narrowing of a parallel composition by composing simplifications of its components, thus avoiding the construction of the actual parallel system. In this paper, we study compositionality for augmented types of Markov chains.

Homogeneous continuous-time Markov chains, Markov chains for short, are among the most important and wide-spread analytical performance models. A Markov chain is given by a graph with nodes representing states and outgoing arrows labelled by exponential rates determining the stochastic behavior of each state. An initial probability vector indicates which states may act as starting ones. Markov chains often come equipped with rewards that are used to measure their performance, such as throughput, utilization, etc. (cf. [1]). In this paper, we focus on state rewards only, and refer to a Markov chain with rewards as a Markov reward chain. A state reward is a number associated to a state, representing the rate at which gain is received while the process resides in the state. Transition (impulse) rewards [1] can similarly be dealt with.

To cope with the ever growing complexity of systems, several performance modeling techniques have been developed to support the compositional generation of Markov reward chains. This includes stochastic process algebras [2,3], (generalized) stochastic Petri nets [4,5], probabilistic I/O automata [6,7], stochastic automata networks [8], etc. The compositional modeling enables composing a bigger system from several smaller components. The size of the state space of the resulting system is in the range of the product of the sizes of the constituent state spaces. Hence, compositional modeling usually suffers from state space explosion.

In the process of compositional modeling, performance evaluation techniques produce intermediate constructs that are typically extensions of Markov chains featuring transitions with communication labels [2-8]. In the final modeling phase, all labels are discarded and communication transitions are assigned instantaneous behavior. The models that we consider here are intended to support direct performance analysis of systems that exhibit both stochastic and instantaneous behavior. We elaborate this for two simple modeling examples using generalized stochastic Petri nets [4] and Interactive Markov Chains [2]. 

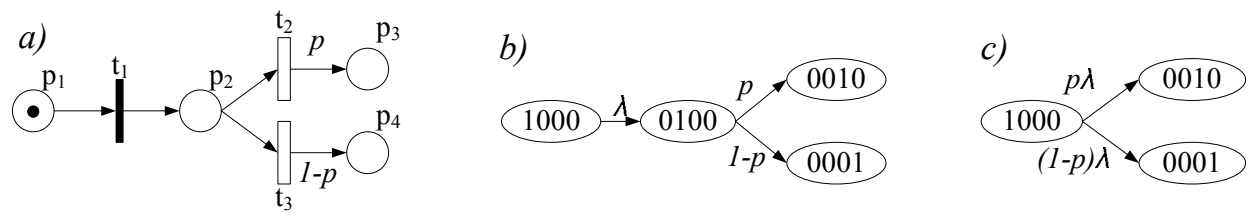

Fig. 1. a) A generalized stochastic Petri net, b) its extended reachability graph, and c) the derived Markov chain.

Example 1 Fig. 1a) depicts an example of a generalized stochastic Petri net with its corresponding reachability graph in Fig. 1b). The graph contains the markings of the only token placed initially in $\mathrm{p}_{1}$. The vanishing marking is 0100 because of the enabled immediate transitions $t_{2}$ and $t_{3}$ with probabilities $p$ and $1-p$, respectively. The Markov chain in Fig. 1c) is obtained by a reduction procedure that splits the incoming normal rate $\lambda$ according the probabilities of the vanishing marking into two rates $p \lambda$ and $(1-p) \lambda$ that reach the final markings 0010 and 0001, respectively.

a)

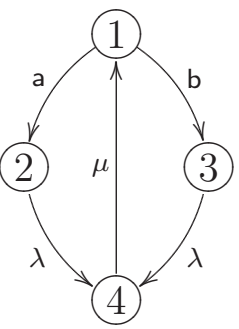

b)

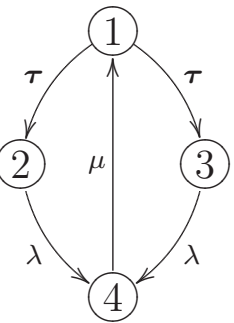

c) 1

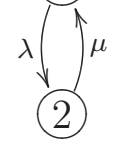

Fig. 2. a) An Interactive Markov Chain, b) the intermediate model with $\boldsymbol{\tau}$-transitions, and c) the induced Markov chain.

Next, consider the Interactive Markov Chain depicted in Fig. 2a). Assuming that the system is closed, the transitions labeled by $\mathrm{a}$ and $\mathrm{b}$ are renamed into the instantaneous transition $\boldsymbol{\tau}$ and an equivalent model is obtained. This intermediate transition system is depicted in Fig. 2b). Now, assume that the process in Fig. 2b) starts from state 1. There it exhibits classical non-determinism, i.e., the probability of taking the $\boldsymbol{\tau}$-transitions is unspecified. Note, however, that the process has the same behavior in states 2 and 3. No matter which transition is taken from state 1 , after performing an instantaneous $\boldsymbol{\tau}$-transition and delaying exponentially with rate $\lambda$, the process enters state 4. According to a bisimulation-based reduction procedure the instantaneous transitions are eliminated and the performance of the process in Fig. 2b) is considered to be the performance of the Markov chain in Fig. 2c).

In both cases in Example 1 the intermediate models depicted in Fig. 1b) and Fig. 2b) are treated only on syntactic level and their performance is defined to be the performance of the final Markov chains from Fig. 1c) and Fig. 2c). Here, we wish to treat the intermediate models as stochastic processes with well-defined notion of performance, which corresponds to the performance of the resulting Markov chain. Markov reward chains with fast transitions were 
introduced in [9-11] to formalize stochastically the elimination of immediate transitions and the vanishing markings of Fig. 1b). They are extensions of the standard Markov reward chains with transitions decorated with a realvalued linear parameter. To capture the intuition that the labeled transitions are instantaneous, a limit for the parameter to infinity is taken. The resulting process is a generalization of the standard Markov reward chain that can perform infinitely many transitions in a finite amount of time. This model was initially studied in $[12,13]$ without rewards, and it is called a (stochastically) discontinuous Markov reward chain. The process exhibits stochastic discontinuity and it is often considered pathological. However, as shown in $[13,14,5]$, it proves very useful for the explanation of results. To deal with the nondeterministic case of Fig. 2b), Markov reward chains with silent transitions were introduced, which generalize Markov reward chains with fast transitions by leaving the linear parameter unspecified.

Here, we consider discontinuous Markov reward chains, Markov reward chains with fast transitions, and Markov reward chains with silent transitions. To summarize, these three models are intimately related: Markov reward chains with fast and silent transitions are used for modeling, but some notions for these processes are expressed asymptotically in terms of discontinuous Markov reward chains. A limiting process of a Markov reward chain with fast transitions is a discontinuous Markov reward chain; a Markov reward chain with silent transitions is identified with an equivalence class of a relation $\sim$ on Markov reward chains with fast transitions relating chains with the 'same shape of fast transitions'. We define parallel composition of all models in vein of standard Markov reward chains using Kronecker products and sums [15].

As already mentioned, compositional modeling may lead to state space explosion. Current analytical and numerical methods can efficiently handle Markov reward chains with millions of states $[16,17]$. However, they only alleviate the problem and many real world problems still cannot be feasibly solved. Several aggregation techniques have been proposed to reduce the state space of Markov reward chains. Ordinary lumping is the most prominent one $[18,15]$. The method partitions the state space into partition classes. In each class, the states exhibit equivalent behavior for transiting to other classes, i.e., the cumulative probability of transiting to another class is the same for every state of the class. If non-trivial lumping exists, i.e., at least one partition contains more than one state, then the method produces a smaller Markov chain that retains the performance characteristics of the original one. For example, the expected reward rate at a given time is the same for the original as for the reduced, so-called lumped, process.

Another lumping-based method is exact lumping $[19,15]$. This method requires that each partition class of states has the same cumulative probability of transiting to every state of another class and, moreover, each state in the 
class has the same initial probability. The gain of exact lumping is that the probabilities of the original process can be computed for a special class of initial probability vectors by using the lumped Markov reward chain only.

A preliminary treatment of relational properties of lumping-based aggregations of Markov chains has been given in [20]. It has been shown that the notion of exact lumping is not transitive, i.e., there are processes which have exactly lumped versions that can be non-trivially exactly lumped again, but the original process cannot be exactly lumped directly to the resulting process. On the other hand, ordinary lumping of Markov reward chains is transitive and, moreover, it has a property of strict confluence. Strict confluence means that whenever a process can be lumped using two different partitions, there is always a smaller process to which the lumped processes can lump to.

Coming back to our models of interest, ordinary lumping is defined for discontinuous Markov reward chains in [9-11]. It is an extension of the standard lumping method that accounts for instantaneous states as well. A lumping method is also defined for Markov reward chains with fast transitions, referred to as $\tau$-lumping [9-11]. The general idea is that two states can be lumped together whenever they can be lumped in the asymptotic discontinuous Markov reward chain, when the parameter tends to infinity. The following commuting diagram gives the agreement of ordinary and $\tau$-lumping. The diagram also shows that $\tau$-lumping is a proper extension of ordinary lumping for standard Markov reward chains.

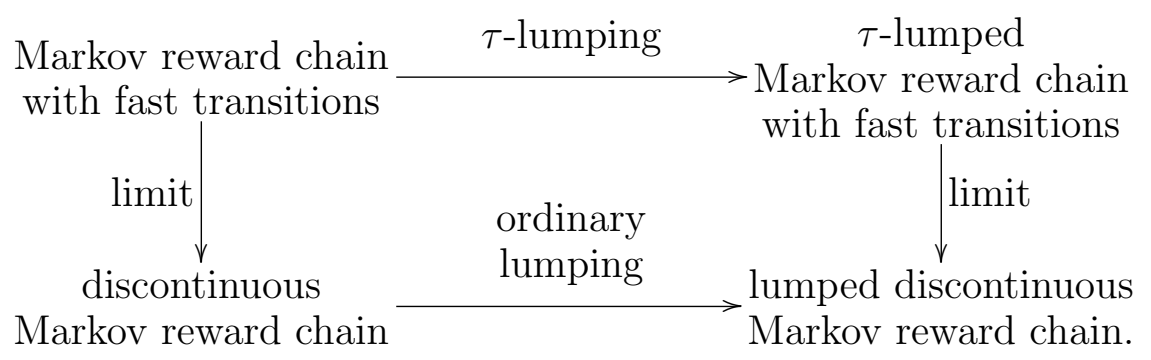

For Markov reward chains with silent transitions, a lifting of $\tau$-lumping to the $\sim$ - equivalence classes is proposed, referred to as $\tau_{\sim}$-lumping [9-11]. More precisely, a partition is a $\tau_{\sim}$-lumping of a Markov reward chain with silent transitions, if it is a $\tau$-lumping for every Markov reward chain with fast transitions in its $\sim$-equivalence class and the $\tau$-lumped process does not depend on the choice of the representative. This situation is depicted in the following figure. 

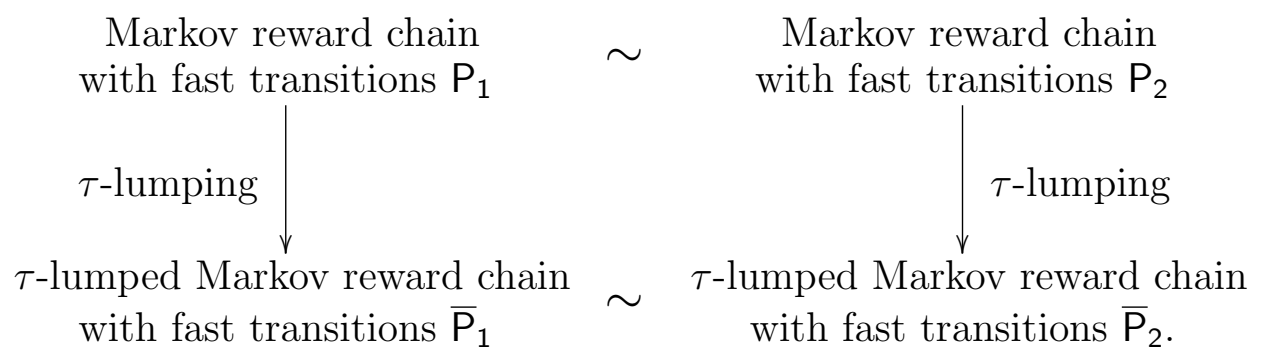

In addition, $[10,11]$ study an aggregation method by reduction that eliminates the stochastic discontinuity and reduces a discontinuous Markov reward chain to a Markov reward chain. The reduction method is an extension of a wellknown method in perturbation theory $[21,22,13]$. Its advantage is the ability to split states. The lumping method, in contrast, provides more flexibility: also states that do not exhibit discontinuous behavior can be aggregated. The reduction-based aggregation straightforwardly extends to $\tau$-reduction of Markov reward chains with fast transitions [10,11]:

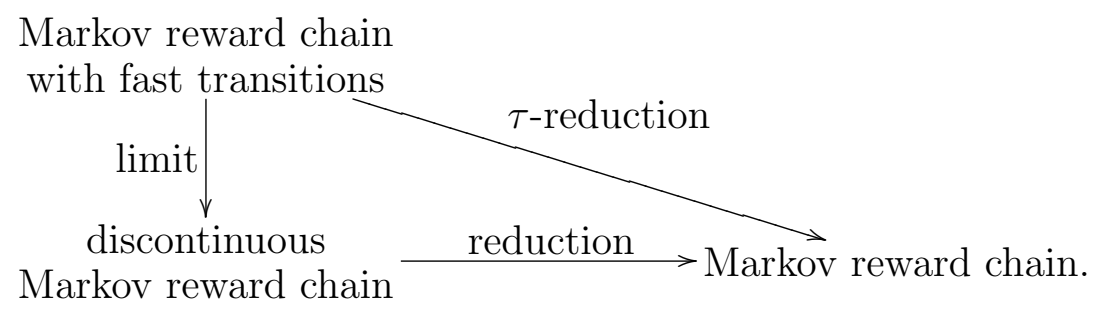

In the case of Markov reward chains with silent transitions, a direct lifting of the $\tau$-reduction to equivalence classes does not aggregate many processes, as most of the time the reduced process depends on the actual fast transitions $[10,11]$. In an attempt to remedy the effect of the fast transitions, we combine $\tau$-reduction and standard ordinary lumping for Markov reward chains to obtain $\tau_{\sim}$-reduction as depicted below. ${ }^{1}$

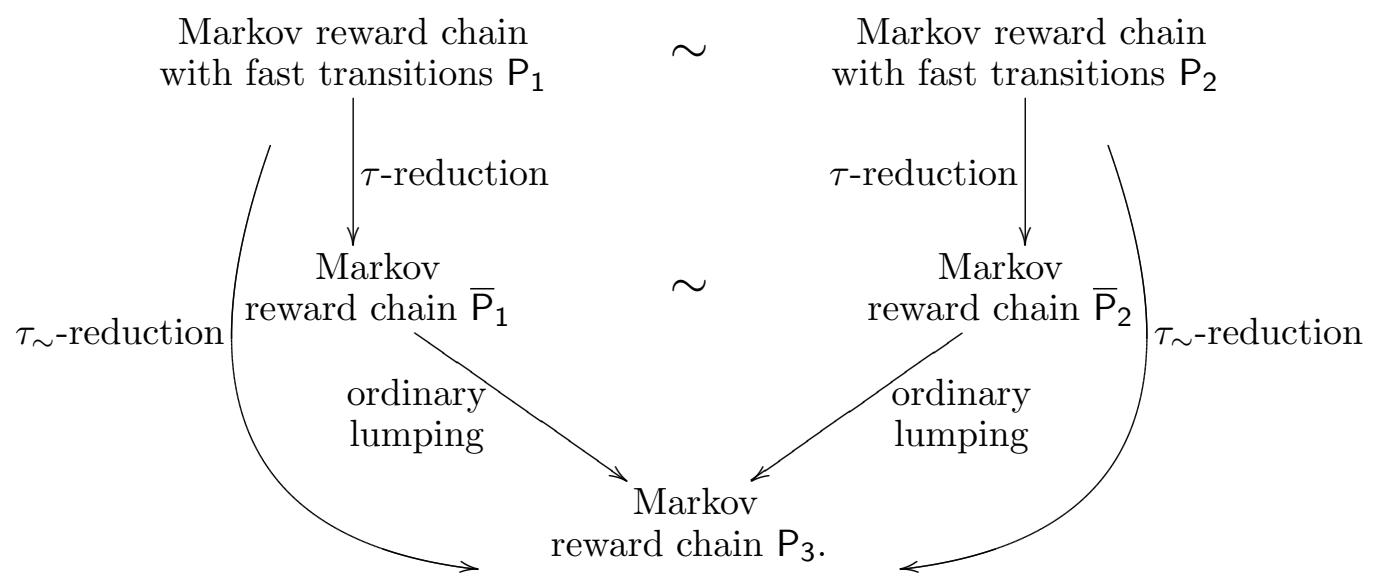

$\overline{1}$ The method is called total $\tau_{\sim}$-reduction in $[10,11]$, since there more $\tau_{\sim}$-reduction methods are considered. 
Hence, a Markov reward chain with silent transitions can be $\tau_{\sim}$-reduced if all Markov reward chains with fast transitions in its equivalence class $\tau$-reduce to Markov reward chains that can be ordinary lumped to the same Markov reward chain.

Both the lumping-based and the reduction-based aggregation method induce semantic relations. Namely, for two processes $P$ and $\bar{P}$, we say that $P \geqslant \bar{P}$ if $\bar{P}$ is an aggregated version of $P$. In our initial study [23], we investigated the properties of these relations for discontinuous Markov reward chains and Markov reward chains with fast transitions. Here we extend the approach to include Markov reward chains with silent transitions as well.

As already mentioned, compositionality is very important as it allows us to aggregate the smaller parallel components first, and then to combine them into the complete aggregated system. This approach can find its way into Markovian model-checkers [24-26] where a huge Markov chain can be (syntactically) decomposed to feasibly-sized components, which can then be aggregated to obtain the aggregated version of the original process. The technique also serves as a validation of existing bisimulation-based reduction methods that treat the intermediate performance models as transition systems. We show that the relations induced by the lumping and reduction methods are indeed preorders, i.e., reflexive and transitive relations. Having all the ingredients in place, we show the compositionality of the aggregation preorders with respect to the defined parallel composition(s). We also show continuity of the parallel composition(s). In short, the parallel operators preserve the diagrams above.

The structure of the rest of the paper is as follows. We start by recalling the three types of Markovian models in Section 2. Section 3 and Section 4 focus on the aggregation methods based on lumping and reduction for each of the models, respectively. Therefore, Section 2 to Section 4 can be understood as an overview of a fairly recent line of research and an introduction to the results that follow. In Section 5, we show that the aggregation methods define preorders on the models. Section 6 contains the main results of the paper: compositionality of the new parallel operators for each type of Markov chains with respect to both aggregation preorders. Section 7 wraps up with conclusions. Throughout the paper we present examples to illustrate the proposed approaches. Whenever we use results from (our) previous work, we state them as propositions with appropriate references. The novel results are stated as theorems.

Notation All vectors are column vectors if not indicated otherwise. By $\mathbf{1}^{n}$ we denote the vector of $n$ 1's; by $0^{n \times m}$ the $n \times m$ zero matrix; by $I^{n}$ the $n \times n$ identity matrix. We omit the dimensions $n$ and $m$ when they are clear from the context. By $A[i, j]$ we denote an element of the matrix $A \in \mathbb{R}^{m \times n}$ assuming $1 \leqslant i \leqslant m$ and $1 \leqslant j \leqslant n$. We write $A \geqslant 0$ when all elements of $A$ 
are non-negative. The matrix $A$ is called stochastic if $A \geqslant 0$ and $A \cdot \mathbf{1}=\mathbf{1}$. By $A^{\top}$ we denote the transpose of $A$.

Let $\mathcal{S}$ be a finite set. A set $\mathcal{P}=\left\{S_{1}, \ldots, S_{N}\right\}$ of $N$ subsets of $\mathcal{S}$ is called a partition of $\mathcal{S}$ if $\mathcal{S}=S_{1} \cup \ldots \cup S_{N}, S_{i} \neq \emptyset$ and $S_{i} \cap S_{j}=\emptyset$ for all $i, j$, with $i \neq j$. The partitions $\{\mathcal{S}\}$ and $\Delta=\{\{i\} \mid i \in \mathcal{S}\}$ are the trivial partitions. Let $\mathcal{P}_{1}=\left\{S_{1}, \ldots, S_{N}\right\}$ be a partition of $\mathcal{S}$ and $\mathcal{P}_{2}=\left\{T_{1}, \ldots, T_{M}\right\}$, in turn, a partition of $\mathcal{P}_{1}$. The composition $\mathcal{P}_{1} \circ \mathcal{P}_{2}$ of the partitions $\mathcal{P}_{1}$ and $\mathcal{P}_{2}$ is a partition of $\mathcal{S}$, given by $\mathcal{P}_{1} \circ \mathcal{P}_{2}=\left\{U_{1}, \ldots, U_{M}\right\}$, where $U_{i}=\cup_{C \in T_{i}} C$.

\section{Markovian Models}

In this section we introduce the Markovian models studied in this paper: discontinuous Markov reward chains as generalizations of standard Markov reward chains where infinitely many transitions can be performed in a finite amount of time; Markov reward chains with fast transitions as Markov reward chains parametrized by a real variable $\tau$; and Markov reward chains with silent transitions as equivalence classes of Markov reward chains with fast transitions with the same structure and unspecified 'speeds' of the fast transitions. The fast transitions explicitly model stochastic behavior, while the silent transitions model non-deterministic internal steps.

\subsection{Discontinuous Markov Reward Chains}

In the standard theory (cf. $[27,28,1]$ ) Markov chains are assumed to be stochastically continuous. This means that the probability is 1 for the process occupying the same state at time $t$ as at time 0 , when $t \rightarrow 0$. As we include instantaneous transitions in our theory [13], this requirement must be dropped. Therefore, we work in the more general setting of discontinuous Markov chains originating from [12].

A discontinuous Markov reward chain is a time-homogeneous finite-state stochastic process with an associated (state) reward structure that satisfies the Markov property. It is completely determined by: (1) a stochastic initial probability row vector that gives the starting probabilities of the process for each state, (2) a transition matrix function $P: \mathbb{R}^{+} \rightarrow \mathbb{R}^{n \times n}$ that defines the stochastic behavior of the transitions at time $t>0$, and (3) a state reward rate vector that associates a number to each state representing the gain of the process while spending time in the state. The transition matrix function gives a stochastic matrix $P(t)$ at any time $t>0$, and has the property $P(t+s)=P(t) \cdot P(s)[27,28]$. It has a convenient characterization independent 
of time [13,29], which allows for the following equivalent definition.

Definition 2 A discontinuous Markov reward chain D is a quadruple $\mathrm{D}=(\sigma, \Pi, Q, \rho)$, where $\sigma$ is a stochastic initial probability row vector, $\rho$ is a state reward vector and $\Pi \in \mathbb{R}^{n \times n}$ and $Q \in \mathbb{R}^{n \times n}$ satisfy the following six conditions: (1) $\Pi \geqslant 0$, (2) $\Pi \cdot \mathbf{1}=\mathbf{1}$, (3) $\Pi^{2}=\Pi$, (4) $\Pi Q=Q \Pi=Q$, (5) $Q \cdot \mathbf{1}=\mathbf{0}$, and (6) $Q+c \Pi \geqslant 0$, for some $c \geqslant 0$. The matrix function $P(t)=\Pi e^{Q t}$ is the transition matrix of $\mathrm{D}$.

We note that the transition matrix uniquely determines the matrices $\Pi$ and $Q$ as given in Definition 2. It is continuous at zero if and only if $\Pi=I$. In this case, $Q$ is a standard generator matrix [13,9]. Otherwise, the matrix $Q$ might contain negative non-diagonal entries. We note that, unlike for standard Markov reward chains, a meaningful graphical representation of discontinuous Markov reward chains when $\Pi \neq I$ is not common. The intuition behind the matrix $\Pi$ is that $\Pi[i, j]$ denotes the probability that a process occupies two states via an instantaneous transition. Therefore, in case of no instantaneous transitions, i.e., when $\Pi=I$, we get a standard (stochastically continuous) Markov reward chain denoted by $\mathrm{M}=(\sigma, Q, \rho)$.

$$
\Pi=\left(\begin{array}{cccc}
\bar{\Pi}_{1} & \ldots & \bar{\Pi}_{M} & \mathbf{0} \\
\Pi_{1} & \ldots & \mathbf{0} & \mathbf{0} \\
\vdots & \ddots & \vdots & \vdots \\
\mathbf{0} & \ldots & \Pi_{M} & \mathbf{0}
\end{array}\right) \quad L=\left(\begin{array}{cccc}
\mu_{1} & \ldots & \mathbf{0} & \mathbf{0} \\
\vdots & \ddots & \vdots & \vdots \\
\mathbf{0} & \ldots & \mu_{M} & \mathbf{0}
\end{array}\right) \quad R=\left(\begin{array}{ccc}
\delta_{1} & \ldots & \delta_{M} \\
\mathbf{1} & \ldots & \mathbf{0} \\
\vdots & \ddots & \vdots \\
\mathbf{0} & \ldots & \mathbf{1}
\end{array}\right)
$$

Fig. 3. The ergodic form of $\Pi$

For every discontinuous Markov reward chain $\mathrm{D}=(\sigma, \Pi, Q, \rho)$, $\Pi$ gets an 'ergodic' form after a suitable renumbering of states [13] as depicted in Fig. 3. Here, for all $1 \leqslant k \leqslant M, \Pi_{k}=\mathbf{1} \cdot \mu_{k}$ and $\bar{\Pi}_{k}=\delta_{k} \cdot \mu_{k}$ for a row vector $\mu_{k}>0$ such that $\mu_{k} \cdot \mathbf{1}=1$ and a vector $\delta_{k} \geqslant 0$ such that $\sum_{k=1}^{M} \delta_{k}=\mathbf{1}$. Then the pair of matrices $(L, R)$ depicted above forms a canonical product decomposition of $\Pi$ (cf. Section 4.1), which is needed for the definition of the reduction-based method of aggregation.

The new numbering induces a partition $\mathcal{E}=\left\{E_{1}, \ldots, E_{M}, T\right\}$ of the state space $\mathcal{S}=\{1, \ldots, n\}$, where $E_{1}, \ldots, E_{M}$ are the ergodic classes, determined by $\Pi_{1}, \ldots, \Pi_{M}$, respectively, and $T$ is the class of transient states, determined by any $\bar{\Pi}_{i}, 1 \leqslant i \leqslant M$. The partition $\mathcal{E}$ is called the ergodic partition. For every ergodic class $E_{k}$, the vector $\mu_{k}$ is the vector of ergodic probabilities. If an ergodic class $E_{k}$ contains exactly one state, then $\mu_{k}=(1)$ and the state is called regular. The vector $\delta_{k}$ contains the trapping probabilities from transient states to the ergodic class $E_{k}$. 
We next discuss the behavior of a discontinuous Markov reward chain $\mathrm{D}=$ $(\sigma, \Pi, Q, \rho)$. It starts in a state with a probability given by the initial probability vector $\sigma$. In an ergodic class with multiple states the process spends a non-zero amount of time switching rapidly (infinitely many times) among the states. The probability that it is found in a specific state of the class is given by the vector of ergodic probabilities. The time the process spends in the class is exponentially distributed and determined by the matrix $Q$. In an ergodic class with a single state the row of $Q$ corresponding to that state has the form of a row in a generator matrix, and $Q[i, j]$ for $i \neq j$ is interpreted as the rate from $i$ to $j$. In a transient state the process spends no time (with probability one) and it immediately becomes trapped in some ergodic class. The process in $i \in T$ can be trapped in $E_{k}$ if and only if the trapping probability $\delta_{k}[i]>0$.

The expected reward (rate) at time $t>0$, notation $R(t)$, is obtained as $R(t)=$ $\sigma P(t) \rho$. It is required in the calculation of the expected accumulated reward up to time $t$, given by $\int_{0}^{t} R(s) d s$. We have that the expected reward remains unchanged if the reward vector $\rho$ is replaced by $\Pi \rho$. To see this, we use that $P(t)=P(t) \Pi$ (cf. [13,11]), so $\sigma P(t) \Pi \rho=\sigma P(t) \rho=R(t)$. Intuitively, the reward in a transient state can be replaced by the sum of the rewards of the ergodic states that it can get trapped in as the process gains no reward while transiting through transient states. The reward of an ergodic state is the sum of the rewards of all states inside its ergodic class weighted according to their ergodic probabilities. This alternative representation of the reward vector alleviates the presentation of some aggregation methods in later sections.

\subsection{Markov Reward Chains with Fast Transitions}

A Markov reward chain with fast transitions is obtained by adding parametrized, so-called fast, transitions to a standard Markov reward chain. The remaining standard transitions are referred to as slow. The behavior of a Markov reward chain with fast transitions is determined by two generator matrices $S$ and $F$, which represent the rates of the slow transitions and the rates (called speeds) of the fast transitions, respectively.

Definition 3 A Markov reward chain with fast transitions $\mathrm{F}=(\sigma, S, F, \rho)$ is a function assigning to each $\tau>0$, the parametrized Markov reward chain

$$
\mathrm{M}_{\tau}=(\sigma, S+\tau F, \rho)
$$

where $\sigma \in \mathbb{R}^{1 \times n}$ is an initial probability vector, $S, F \in \mathbb{R}^{n \times n}$ are two generator matrices, and $\rho \in \mathbb{R}^{n \times 1}$ is the reward vector.

By taking the limit when $\tau \rightarrow \infty$, fast transitions become instantaneous. Then, a Markov reward chain with fast transitions behaves as a discontinuous 
Markov reward chain [13,9-11].

Definition 4 Let $\mathrm{F}=(\sigma, S, F, \rho)$ be a Markov reward chain with fast transitions. The discontinuous Markov reward chain $\mathrm{D}=(\sigma, \Pi, Q, \Pi \rho)$ is the limit of $\mathrm{F}$, where the matrix $\Pi$ is the so-called ergodic projection at zero of $F$, that is $\Pi=\lim _{t \rightarrow \infty} e^{F t}$, and $Q=\Pi S \Pi$. We write $\mathbf{F} \rightarrow_{\infty} \mathrm{D}$.

The initial probability vector is not affected by the limit construction. We will later motivate the choice of using the reward vector $\Pi \rho$ instead of just $\rho$. In addition, we define the ergodic partition of a Markov reward chain with fast transitions to be the ergodic partition of its limit discontinuous Markov reward chain.

To illustrate the relation between the Markov reward chains with fast transitions and discontinuous Markov reward chains we give an example.

Example 5 We depict Markov reward chains with fast transitions as in Fig. 4. The initial probabilities are depicted left above, and the reward rates right above each state. Here, $a, b$, and $c$ are speeds, whereas $\lambda, \mu, \nu$, and $\xi$ are rates of slow transitions. As in the definition, $\tau$ denotes the real parameter.
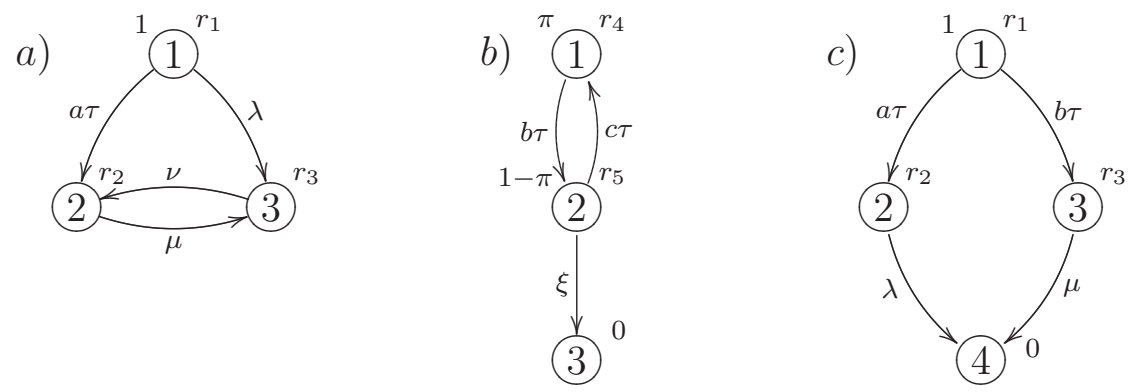

Fig. 4. Markov reward chains with fast transitions

The limit of the Markov reward chain with fast transitions in Fig. 4a) is given by the discontinuous Markov reward chain $\mathrm{D}_{1}=\left(\sigma_{1}, \Pi_{1}, Q_{1}, \rho_{1}\right)$ with:

$$
\sigma_{1}=\left(\begin{array}{lll}
1 & 0 & 0
\end{array}\right) \quad \Pi_{1}=\left(\begin{array}{lll}
0 & 1 & 0 \\
0 & 1 & 0 \\
0 & 0 & 1
\end{array}\right) \quad Q_{1}=\left(\begin{array}{ccc}
0 & -\mu & \mu \\
0 & -\mu & \mu \\
0 & \nu & -\nu
\end{array}\right) \quad \rho_{1}=\left(\begin{array}{l}
r_{2} \\
r_{2} \\
r_{3}
\end{array}\right)
$$

State 1 is transient, whereas states 2 and 3 are regular.

The limit of the Markov reward chain with fast transitions in Fig. 4b) is given 
by $\mathrm{D}_{2}=\left(\sigma_{2}, \Pi_{2}, Q_{2}, \rho_{2}\right)$ with:

$\sigma_{2}=\left(\begin{array}{lll}\pi & 1-\pi & 0\end{array}\right) \Pi_{2}=\left(\begin{array}{ccc}p & q & 0 \\ p & q & 0 \\ 0 & 0 & 1\end{array}\right) \quad Q_{2}=\left(\begin{array}{ccc}-p q \xi-q^{2} \xi & q \xi \\ -p q \xi & -q^{2} \xi & q \xi \\ 0 & 0 & 0\end{array}\right) \quad \rho_{2}=\left(\begin{array}{c}p r_{4}+q r_{5} \\ p r_{4}+q r_{5} \\ 0\end{array}\right)$,

where $p=\frac{c}{b+c}$ and $q=\frac{b}{b+c}$. States 1 and 2 in Fig. 4b) form an ergodic class and state 3 is regular.

Finally, the limit of the process depicted in Fig. 4c) is given by the discontinuous Markov reward chain $\mathrm{D}_{3}=\left(\sigma_{3}, \Pi_{3}, Q_{3}, \rho_{3}\right)$ with:

$$
\sigma_{3}=\left(\begin{array}{llll}
1 & 0 & 0 & 0
\end{array}\right) \Pi_{3}=\left(\begin{array}{cccc}
0 & \frac{a}{a+b} & \frac{b}{a+b} & 0 \\
0 & 1 & 0 & 0 \\
0 & 0 & 1 & 0 \\
0 & 0 & 0 & 1
\end{array}\right) \quad Q_{3}=\left(\begin{array}{cccc}
0 & 0 & 0 & 0 \\
0 & -\lambda & 0 & \lambda \\
0 & 0 & -\mu & \mu \\
0 & 0 & 0 & 0
\end{array}\right) \quad \rho_{3}=\left(\begin{array}{c}
0 \\
r_{2} \\
r_{3} \\
0
\end{array}\right) .
$$

State 1 in Fig. 4c) is transient, whereas states 2, 3, and 4 are regular.

\subsection{Markov Reward Chains with Silent Transitions}

We define a Markov reward chain with silent transitions as a Markov reward chain with fast transitions in which the speeds of the fast transitions are left unspecified. To abstract away from the speeds of the fast transitions we introduce a suitable equivalence relation on Markov reward chains with fast transitions that is induced by the following equivalence relation of matrices.

Definition 6 Two matrices $A, B \in \mathbb{R}^{n \times n}$ have the same shape (also called grammar), notation $A \sim B$, if and only if they have zeros on the same positions. That is,

$$
A \sim B \Longleftrightarrow(\forall i, j)(A[i, j]=0 \Longleftrightarrow B[i, j]=0) .
$$

It is obvious that $\sim$ is an equivalence on matrices of the same order. The abstraction from speeds is achieved by identifying generator matrices of fast transitions with the same shape. Thus, silent transitions are modeled by equivalence classes of $\sim$.

Definition 7 A Markov reward chain with silent transitions $\mathrm{S}$ is a quadruple $\mathrm{S}=(\sigma, S, \mathcal{F}, \rho)$ where $\mathcal{F}$ is an equivalence class of $\sim$ and, for every $F \in \mathcal{F}$, $\mathrm{F}=(\sigma, S, F, \rho)$ is a Markov reward chain with fast transitions. 
We write $\mathbf{F} \in \mathbf{S}$ if $\mathbf{S}=(\sigma, S, \mathcal{F}, \rho)$, and $\mathbf{F}=(\sigma, S, F, \rho)$ with $F \in \mathcal{F}$. Furthermore, we lift the relation $\sim$ to Markov reward chains with fast transitions and write $F \sim F^{\prime}$ if $F, F^{\prime} \in S$. The notion of an ergodic partition is speed independent, i.e., if $F \sim F^{\prime}$, then they have the same ergodic partition, since the ergodic partition depends only on the existence of fast transitions, but not on the actual speeds. Hence we can define the ergodic partition of a Markov reward chain with silent transitions $S$ as the ergodic partition of any Markov reward chain with fast transitions $F$ such that $F \in S$.
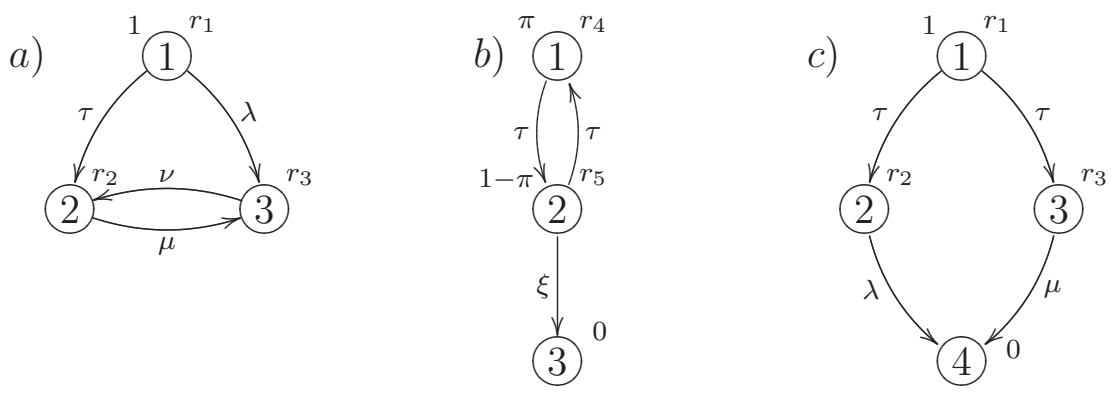

Fig. 5. Markov reward chains with silent transitions

Example 8 We depict Markov reward chains with silent transitions as in Fig. 5, i.e., by omitting the speeds of the fast transitions. The depicted Markov reward chains with silent transitions are induced by the Markov reward chains with fast transitions in Fig. 4.

In Fig. 5, $\tau$ can be understood as a label of internal action transitions, as it is common in transition system modeling and process algebra [30,31]. In this way we formalize the notion of performance analysis for Markov reward chains with non-deterministic internal steps.

\section{$3 \quad$ Aggregation by Lumping}

In this section we present lumping methods for the Markovian models of the previous section originally studied in [9-11]. First, we generalize ordinary lumping of [18] to discontinuous Markov reward chains. Then, we define $\tau$-lumping for Markov reward chains with fast transitions based on ordinary lumping of discontinuous Markov reward chains. Finally, we lift the $\tau$-lumping to $\tau_{\sim}$-lumping of Markov reward chains with silent transitions.

We define aggregation by lumping in terms of matrices. Every partition $\mathcal{P}=\left\{C_{1}, \ldots, C_{N}\right\}$ of $\mathcal{S}=\{1, \ldots, n\}$ can be associated with a so-called collector matrix $V \in \mathbb{R}^{n \times N}$ defined by $V[i, k]=0$ if $i \notin C_{k}, V[i, k]=1$ if $i \in C_{k}$, and vice versa. The $k$-th column of $V$ has 1's for elements corresponding to states in $C_{k}$ and has 0 's otherwise. Note that $V \cdot \mathbf{1}=\mathbf{1}$. A distributor matrix $U \in \mathbb{R}^{N \times n}$ for $\mathcal{P}$ is defined as a matrix $U \geqslant 0$, such that $U V=I^{N}$. To satisfy 
these conditions, the elements of the $k$-th row of $U$, which correspond to states in the class $C_{k}$, sum up to one, whereas the other elements of the row are 0 .

\subsection{Ordinary Lumping}

An ordinary lumping is a partition of the state space of a discontinuous Markov reward chain into classes such that the states that are lumped together have equivalent behavior for transiting to other classes and, additionally, have the same reward.

Definition 9 A partition $\mathcal{L}$ of $\{1, \ldots, n\}$ is an ordinary lumping, or lumping for short, of a discontinuous Markov reward chain $\mathrm{D}=(\sigma, \Pi, Q, \rho)$ if and only if the following hold: (1) $V U \Pi V=\Pi V$, (2) $V U Q V=Q V$, and (3) $V U \rho=\rho$, where $V$ is the collector matrix and $U$ is any distributor matrix for $\mathcal{L}$.

The lumping conditions only require that the rows of $\Pi V$ (respectively $Q V$ and $\rho$ ) that correspond to the states of the same partition class are equal. We have the following property.

Proposition $10([\mathbf{9 - 1 1}])$ Let $\mathrm{D}=(\sigma, \Pi, Q, \rho)$ be a discontinuous Markov reward chain and let $\mathcal{L}$ be its ordinary lumping. Define (1) $\bar{\sigma}=\sigma V$, (2) $\bar{\Pi}=$ $U \Pi V$, (3) $\bar{Q}=U Q V$ and (4) $\bar{\rho}=U \rho$, for the collector matrix $V$ of $\mathcal{L}$ and any distributor $U$. Then $\overline{\mathrm{D}}=(\bar{\sigma}, \bar{\Pi}, \bar{Q}, \bar{\rho})$ is a discontinuous Markov reward chain. Moreover, $\bar{P}(t)=U P(t) V$ where $\bar{P}(t)$ and $P(t)$ are the transition matrices of $\overline{\mathrm{D}}$ and $\mathrm{D}$, respectively.

Definition 11 If the conditions of Proposition 10 are satisfied, then $\mathrm{D}=$ $(\sigma, \Pi, Q, \rho)$ lumps to $\overline{\mathrm{D}}=(\bar{\sigma}, \bar{\Pi}, \bar{Q}, \bar{\rho})$, called the lumped discontinuous Markov reward chain with respect to $\mathcal{L}$. We write $\mathrm{D} \stackrel{\mathcal{L}}{\rightarrow} \overline{\mathrm{D}}$.

It can readily be seen that neither the definition of a lumping, nor the definition of the lumped process depends on the choice of a distributor matrix $U$. For example, if $V U Q V=Q V$, then $V U^{\prime} Q V=V U^{\prime} V U Q V=V U Q V=Q V$, for any other distributor $U^{\prime}$. In the continuous case, when $\Pi=I$ we have $\bar{\Pi}=I$, so $\bar{Q}$ is a generator matrix and our notion of ordinary lumping coincides with the standard definition $[18,32]$. The expected reward is preserved by ordinary lumping, since:

$$
\bar{R}(t)=\sigma V U P(t) V U \rho=\sigma P(t) V U \rho=\sigma P(t) \rho=R(t) .
$$

Similarly, as in [18], one can show that other performance measures are also preserved by lumping. To illustrate the method we lump the discontinuous Markov reward chains of Example 5. 
Example 12 It is directly checked that the lumping condition holds for the discontinuous Markov reward chains $\mathrm{D}_{1}$ and $\mathrm{D}_{2}$ of Example 5 for the same partition $\{\{1,2\},\{3\}\}$. The discontinuous Markov reward chain $\mathrm{D}_{3}$ can be lumped only if $\lambda=\mu$ and $r_{2}=r_{3}$ using the partition $\{\{1,2,3\},\{4\}\}$. For the lumped version $\overline{\mathrm{D}}_{1}=\left(\bar{\sigma}_{1}, \bar{\Pi}_{1}, \bar{Q}_{1}, \bar{\rho}_{1}\right)$ of $\mathrm{D}_{1}$ we obtain:

$$
V_{1}=\left(\begin{array}{ll}
1 & 0 \\
1 & 0 \\
0 & 1
\end{array}\right) \quad \bar{\sigma}_{1}=\left(\begin{array}{ll}
1 & 0
\end{array}\right) \quad \bar{\Pi}_{1}=\left(\begin{array}{ll}
1 & 0 \\
0 & 1
\end{array}\right) \quad \bar{Q}_{1}=\left(\begin{array}{cc}
-\mu & \mu \\
\nu & -\nu
\end{array}\right) \quad \bar{\rho}_{1}=\left(\begin{array}{l}
r_{2} \\
r_{3}
\end{array}\right) .
$$

The lumped process $\overline{\mathrm{D}}_{1}$ is a standard Markov reward chain as $\bar{\Pi}_{1}$ is the identity matrix.

For the lumped version $\overline{\mathrm{D}}_{2}=\left(\bar{\sigma}_{2}, \bar{\Pi}_{2}, \bar{Q}_{2}, \bar{\rho}_{2}\right)$ of $\mathrm{D}_{2}$ we obtain:

$$
V_{2}=\left(\begin{array}{ll}
1 & 0 \\
1 & 0 \\
0 & 1
\end{array}\right) \quad \bar{\sigma}_{2}=\left(\begin{array}{ll}
1 & 0
\end{array}\right) \bar{\Pi}_{2}=\left(\begin{array}{ll}
1 & 0 \\
0 & 1
\end{array}\right) \bar{Q}_{2}=\left(\begin{array}{cc}
-q \xi & q \xi \\
0 & 0
\end{array}\right) \bar{\rho}_{2}=\left(\begin{array}{c}
p r_{4}+q r_{5} \\
0
\end{array}\right),
$$

where $p=\frac{c}{b+c}$ and $q=1-p$. Again, the result is a standard Markov reward chain.

If we assume that $\lambda=\mu$ and $r_{2}=r_{3}$, then the lumped version of $\mathrm{D}_{3}$ is $\overline{\mathrm{D}}_{3}=\left(\bar{\sigma}_{3}, \bar{\Pi}_{3}, \bar{Q}_{3}, \bar{\rho}_{3}\right)$ with:

$$
V_{3}=\left(\begin{array}{ll}
1 & 0 \\
1 & 0 \\
1 & 0 \\
0 & 1
\end{array}\right) \quad \bar{\sigma}_{3}=\left(\begin{array}{ll}
1 & 0
\end{array}\right) \bar{\Pi}_{3}=\left(\begin{array}{ll}
1 & 0 \\
0 & 1
\end{array}\right) \bar{Q}_{3}=\left(\begin{array}{cc}
-\lambda & \lambda \\
0 & 0
\end{array}\right) \bar{\rho}_{3}=\left(\begin{array}{l}
r_{2} \\
0
\end{array}\right),
$$

which is again a standard Markov reward chain.

The lumped processes are depicted in Fig. 6.
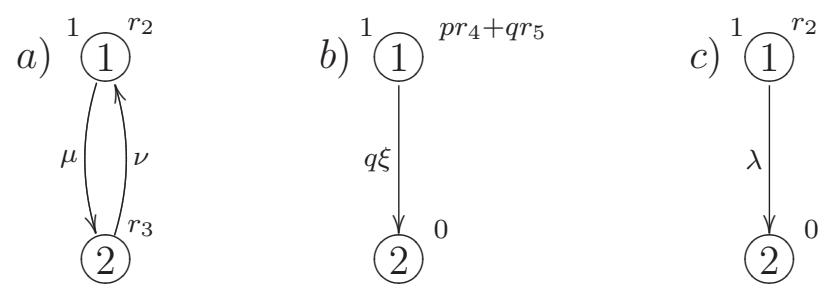

Fig. 6. Aggregated Markov reward chains with fast transitions of Fig. 4 
The notion of $\tau$-lumping is based on ordinary lumping for discontinuous Markov reward chains. The aim is that the limit of a $\tau$-lumped Markov reward chain with fast transitions is an ordinary lumped version of the limit of the original Markov reward chain with fast transitions.

Definition 13 A partition $\mathcal{L}$ of the state space of a Markov reward chain with fast transitions $\mathrm{F}$ is called a $\tau$-lumping, if it is an ordinary lumping of its limiting discontinuous Markov reward chain $\mathrm{D}$ with $\mathrm{F} \rightarrow_{\infty} \mathrm{D}$.

Note that since we defined the reward of the limit by $\Pi \rho$, a $\tau$-lumping may identify states with different rewards.

Like for ordinary lumping, we define the $\tau$-lumped process by multiplying $\sigma, S$, $F$ and $\rho$ with a collector matrix and a distributor matrix. However, unlike for ordinary lumping, not all distributors are allowed. In [9-11] a class of special distributors, called $\tau$-distributors, is provided that yield a $\tau$-lumped process.

Definition 14 Let $\mathrm{D}=(\sigma, \Pi, Q, \rho)$ be a discontinuous Markov reward chain. Let $V$ be a collector corresponding to a partition of the state space of this chain. A matrix $W$ is a $\tau$-distributor for $V$ if and only if (1) it is a distributor for $V$, (2) $\Pi V W \Pi=\Pi V W$, and (3) the entries of $W$ corresponding to states in classes of transient states are positive.

$A$-distributor for a partition of a Markov reward chain with fast transitions is any $\tau$-distributor for the same partition of its limiting discontinuous Markov reward chain.

Remark 15 An alternative, explicit definition of the $\tau$-distributors can be found in [9-11]. We note here that the class of $\tau$-distributors given by Definition 14 depends on two sets of parameters. Namely, after suitable renumbering, any $\tau$-distributor $W$ can be written as $W=\left(\begin{array}{cc}W(\alpha) & \mathbf{0} \\ \mathbf{0} & W(\beta)\end{array}\right)$, where $W(\alpha)$ is a distributor for the classes containing ergodic states and $W(\beta)$ is a distributor for the classes of transient states. As the notation suggests, the distributor $W(\alpha)$ depends on a set of parameters $\alpha$ and the distributor $W(\beta)$ is determined by a set of parameters $\beta$. To explicitly state this dependence we may write $W_{\alpha, \beta}$ for a $\tau$-distributor depending on the parameter sets $\alpha$ and $\beta$. By the alternative definition of $\tau$-distributors we can also establish the existence of a $\tau$-distributor for any $\tau$-lumping.

Having defined $\tau$-distributors, we can now explicitly define a $\tau$-lumped process.

Definition 16 Let $\mathrm{F}=(\sigma, S, F, \rho)$ and let $\mathcal{L}$ be a lumping with a collector matrix $V$, and a corresponding $\tau$-distributor $W$. The $\tau$-lumped Markov reward chain with fast transitions $\overline{\mathrm{F}}=(\bar{\sigma}, \bar{S}, \bar{F}, \bar{\rho})$ is defined as $\bar{\sigma}=\sigma V, \bar{S}=$ 
$W S V, \bar{F}=W F V, \bar{\rho}=W \rho$. We say that $\mathrm{F} \tau$-lumps to $\overline{\mathrm{F}}$ with respect to $W$ and write $\mathrm{F} \stackrel{\mathcal{L}}{\rightarrow} \overline{\mathrm{F}}$. We write $\mathrm{F} \stackrel{\mathcal{L}}{\sim} \overline{\mathrm{F}}$ if $\mathrm{F} \stackrel{\mathcal{L}}{\sim} \overline{\mathrm{F}}$ for some $\tau$-distributor $W$.

In general, when lumping $\mathrm{F}$ using a collector $V$ and a distributor $U, U S V$ and $U F V$ are not uniquely determined, i.e., they depend on the choice of the distributor. The restriction to $\tau$-distributors does not change this. Subsequently, the $\tau$-lumped process depends on the choice of the $\tau$-distributor. In order to

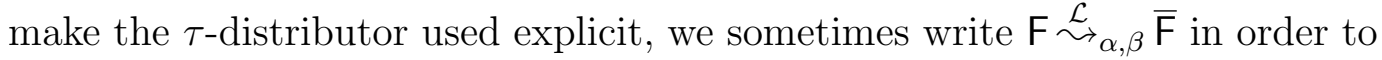
emphasize the parameter sets such that $W=W_{\alpha, \beta}$.

The motivation for restricting to $\tau$-distributors, despite that they do not ensure a unique $\tau$-lumped process, is that all $\tau$-lumped processes are equivalent in the limit. This is stated in the following proposition, which gives the precise connection of ordinary lumping and $\tau$-lumping.

Proposition 17 ([9-11]) The following diagram commutes

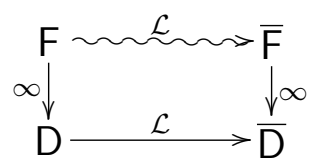

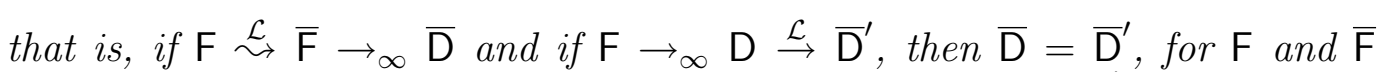
Markov reward chains with fast transitions, and $\mathrm{D}, \overline{\mathrm{D}}$, and $\overline{\mathrm{D}}^{\prime}$ discontinuous Markov reward chains.

Moreover, the $\tau$-lumped processes that originate from the same Markov reward chain with fast transitions become exactly the same, once all fast transitions are eliminated $[10,11]$.

Example 18 The $\tau$-distributors (cf. [10,11] for the explicit form of the $\tau$ distributors) for the Markov reward chains with fast transitions depicted in Fig. 4a), Fig. 4b), and Fig. 4c) are

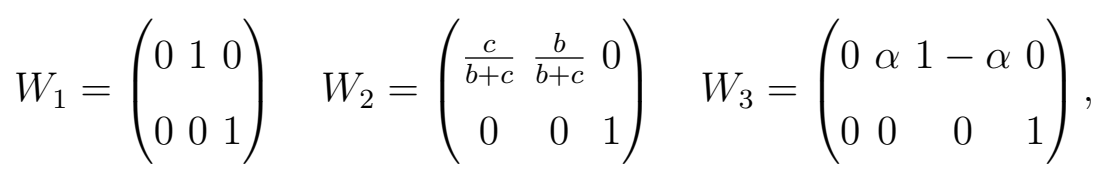

for some $0 \leq \alpha \leq 1$, respectively. Again we assume that $\lambda=\mu$ and $r_{2}=r_{3}$ for the Markov reward chain with fast transitions in Fig. 4c) to be $\tau$-lumpable. It is directly checked that the $\tau$-lumped processes correspond to the lumped Markov reward chains from Example 12 depicted in Fig. 6 as expected by Proposition 17. 
We lift $\tau$-lumping to equivalence classes of $\sim$ to obtain $\tau_{\sim}$-lumping for Markov reward chains with silent transitions. Intuitively, a partition is a $\tau_{\sim}$-lumping of $\mathbf{S}$, if it is a $\tau$-lumping for every $\mathbf{F} \in \mathbf{S}$ and, moreover, the limit of the $\tau$-lumped process of $\mathrm{F}$ does not depend on the parameters chosen for the $\tau$ distributor. Recall that the parameter set $\alpha$ affects ergodic states, whereas the parameter set $\beta$ affects only transient states.

Definition 19 Let $\mathrm{S}$ be a Markov reward chain with silent transitions and let $\mathcal{L}$ be its partition. Then $\mathcal{L}$ is a $\tau_{\sim}$-lumping if and only if it is a $\tau$-lumping for every Markov reward chain with fast transitions $\mathrm{F} \in \mathrm{S}$ and, moreover, for

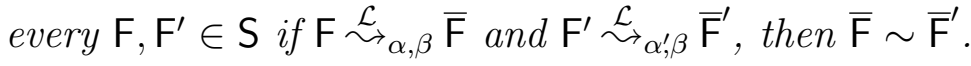

The motivation behind the use of the same parameter set $\beta$ in Definition 19 is that there may be slow transitions originating from transient states which will depend on $\beta$ in the lumped process. If we do not restrict to the same parameter set $\beta$, then $\tau_{\sim}$-lumpings will only exist in rare cases in which transient states have no slow transitions. We refer to $[10,11]$ for details.

Now we can define a $\tau_{\sim}$-lumped process which is unique for a given $\tau_{\sim}$-lumping $\mathcal{L}$ and a parameter set $\beta$.

Definition 20 Let $\mathrm{S}$ be a Markov reward chain with silent transitions and $\mathcal{L}$ its $\tau_{\sim}$-lumping. Let $\mathrm{F} \in \mathrm{S}$ be such that $\mathrm{F} \stackrel{\mathcal{L}}{\sim}{ }_{\alpha, \beta} \overline{\mathrm{F}}$ and let $\overline{\mathrm{S}}$ be the Markov reward chain with silent transitions with $\overline{\mathrm{F}} \in \overline{\mathrm{S}}$. Then $\mathrm{S} \tau_{\sim}$-lumps to $\overline{\mathrm{S}}$, with respect to $\mathcal{L}$ and $\beta$, notation $\mathbf{S} \stackrel{\mathcal{L}}{\rightarrow} \overline{\mathrm{S}}$. We write $\mathbf{S} \stackrel{\mathcal{L}}{\sim} \overline{\mathrm{S}}$ if $\mathbf{S} \stackrel{\mathcal{L}}{\rightarrow} \overline{\mathrm{S}}$ for some parameter set $\beta$.

Example 21 From the lumped and $\tau$-lumped versions of Example 12 and Example 18, respectively, we conclude that the Markov reward chain with silent transitions depicted in Fig. 5a) can be $\tau_{\sim}$-lumped to the Markov reward chain $\overline{\mathrm{D}}_{1}$ of Example 12 depicted in Fig. 6a) as every representative Markov reward chain with fast transitions lumps to this process. The Markov reward chain with silent transitions depicted in Fig. 5b) cannot be $\tau_{\sim}$-lumped as the $\tau$-lumped versions of the representative Markov reward chains with fast transitions always depend on the parameters $b$ and $c$ and no further lumping is possible. Finally, if we assume that $\lambda=\mu$ and $r_{2}=r_{3}$ for the Markov reward chain with silent transitions depicted in Fig. 5c), then it can be $\tau_{\sim}$-lumped to the Markov reward chain $\overline{\mathrm{D}}_{3}$ of Example 12 depicted in Fig. 6c). 


\section{Aggregation by Reduction}

Reduction is a specific aggregation method for transforming a discontinuous Markov chain into a standard Markov chain, originally studied in $[21,22,13]$. Extended to reward processes, the method reduces a discontinuous Markov reward chain to a Markov reward chain by eliminating instantaneous states, while retaining the behavior of the regular states. In the same spirit, we define reduction methods that reduce Markov reward chains with fast and silent transitions to Markov reward chains following [10,11], called $\tau$-reduction and $\tau_{\sim}$-reduction, respectively.

\subsection{Reduction}

The reduction-based aggregation method masks the stochastic discontinuity of a discontinuous Markov reward chain and transforms it into a Markov reward chain $[21,13,10,11]$. The underlying idea is to abstract away from the behavior of individual states in an ergodic class. The method is based on the notion of a canonical product decomposition.

Definition 22 Let $\mathrm{D}=(\sigma, \Pi, Q, \rho)$ and assume that $\operatorname{rank}(\Pi)=M$, i.e., that there are $M$ ergodic classes. A canonical product decomposition of $\Pi$ is a pair of matrices $(L, R)$ with $L \in \mathbb{R}^{M \times n}$ and $R \in \mathbb{R}^{n \times M}$ such that $L \geqslant 0, R \geqslant 0$, $\operatorname{rank}(L)=\operatorname{rank}(R)=M, L \cdot \mathbf{1}=\mathbf{1}$, and $\Pi=R L$.

A canonical product decomposition always exists and it can be constructed from the ergodic form of $\Pi$ (see Fig. 3). Moreover, it can be shown that any other canonical product decomposition is permutation equivalent to this one. Since a canonical product decomposition $(L, R)$ of $\Pi$ is a full-rank decomposition, and since $\Pi$ is idempotent, we also have that $L R=I^{M}$. Thus, we have $L \Pi=L R L=L$ and $\Pi R=R L R=R$. Next, we present the reduction method.

Definition 23 For a discontinuous Markov reward chain $\mathrm{D}=(\sigma, \Pi, Q, \rho)$, the reduced Markov reward chain $\mathrm{M}=(\bar{\sigma}, \bar{Q}, \bar{\rho})$ is given by $\bar{\sigma}=\sigma R, \bar{Q}=L Q R$ and $\bar{\rho}=L \rho$, where $(L, R)$ is a canonical product decomposition of $\Pi$. We write $\mathrm{D} \rightarrow{ }_{r} \mathrm{M}$.

If $\bar{P}(t)$ and $P(t)$ are the transition matrices of the reduced and the original chain, respectively, then one can show that $\bar{P}(t)=L P(t) R$, see $[13,22]$.

The reduced process is unique up to a permutation of the states, since the canonical product decomposition is. The states of the reduced process are given by the ergodic classes of the original process, while the transient states 
are 'ignored'. Intuitively, the transient states are split probabilistically between the ergodic classes according to their trapping probabilities. In case a transient state is also an initial state, its initial probability is split according to its trapping probabilities. The reward rate is calculated as the sum of the individual reward rates of the states of the ergodic class weighted by their ergodic probabilities. Like lumping, the reduction also preserves the expected reward rate at time $t$ :

$$
\bar{R}(t)=\sigma R L P(t) R L \rho=\sigma \Pi P(t) \Pi \rho=\sigma P(t) \rho=R(t)
$$

In case the original process has no stochastic discontinuity, i.e., $\Pi=I$, the reduced process is equal to the original.

\section{2 $\tau$-Reduction}

We now define a reduction-based aggregation method called $\tau$-reduction. It aggregates a Markov reward chain with fast transitions to an asymptotically equivalent Markov reward chain.

Definition 24 A Markov reward chain with fast transitions $\mathrm{F}=(\sigma, S, F, \rho)$ $\tau$-reduces to the Markov reward chain $\mathrm{M}=(\bar{\sigma}, \bar{Q}, \bar{\rho})$, given by (1) $\bar{\sigma}=\sigma R$, (2) $\bar{Q}=L S R$, and (3) $\bar{\rho}=L \rho$, where $\mathrm{F} \rightarrow_{\infty}(\sigma, \Pi, Q, \Pi \rho)$ and $(L, R)$ is a canonical product decomposition of $\Pi$. When $\mathrm{F} \tau$-reduces to $\mathrm{M}$, we write $\mathrm{F} \sim_{r} \mathrm{M}$.

The following simple property relates $\tau$-reduction to reduction. It holds since $L Q R=L \Pi S \Pi R=L S R$ and $L \Pi \rho=L \rho$.

Proposition 25 ([10,11]) The following diagram commutes

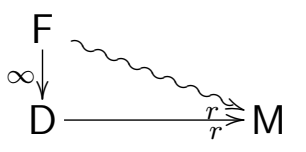

that is, if $\mathrm{F} \sim_{r} \mathrm{M}$ and $\mathrm{F} \rightarrow_{\infty} \mathrm{D} \rightarrow_{r} \mathrm{M}^{\prime}$, then $\mathrm{M}=\mathrm{M}^{\prime}$, for $\mathrm{F}$ a Markov reward chain with fast transitions, D a discontinuous Markov reward chain and $\mathrm{M}$ and $\mathrm{M}^{\prime}$ (continuous) Markov reward chains.

Example 26 The aggregation by $\tau$-reduction reduces the Markov reward chains with fast transitions of Fig. 4a) and Fig. 4b) to the Markov reward chains $\overline{\mathrm{D}}_{1}$ and $\overline{\mathrm{D}}_{2}$ of Example 12, respectively, as in the case of $\tau$-lumping. However, by using $\tau$-reduction the Markov reward chain with fast transitions depicted in Fig. 4c) can be directly aggregated to a Markov reward chain without any assumptions. The canonical decompositions for the Markov reward 
chains with fast transitions depicted in Figs. 4a), b), and c) are:

$$
\begin{aligned}
& L_{1}=\left(\begin{array}{lll}
0 & 1 & 0 \\
0 & 0 & 1
\end{array}\right) \quad R_{1}=\left(\begin{array}{ll}
1 & 0 \\
1 & 0 \\
0 & 1
\end{array}\right) \quad L_{2}=\left(\begin{array}{ccc}
\frac{c}{b+c} & \frac{b}{b+c} & 0 \\
0 & 0 & 1
\end{array}\right) \quad R_{2}=\left(\begin{array}{ll}
1 & 0 \\
1 & 0 \\
0 & 1
\end{array}\right) \\
& L_{3}=\left(\begin{array}{llll}
0 & 1 & 0 & 0 \\
0 & 0 & 1 & 0 \\
0 & 0 & 0 & 1
\end{array}\right) \quad R_{3}=\left(\begin{array}{ccc}
\frac{a}{a+b} & \frac{b}{a+b} & 0 \\
1 & 0 & 0 \\
0 & 1 & 0 \\
0 & 0 & 1
\end{array}\right) .
\end{aligned}
$$

The Markov reward chain with fast transitions of Fig. 4c) reduces to the Markov reward chain depicted in Fig. 7. Note that the initial probability vector is split according to the transient probabilities.

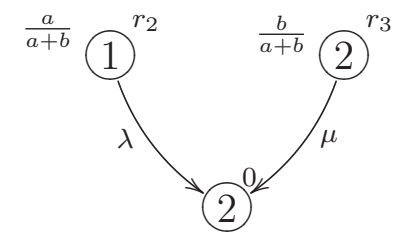

Fig. 7. $\tau$-reduced Markov reward chain with fast transitions of Fig. 4c)

\section{$4.3 \tau_{\sim}$-Reduction}

By combining $\tau$-reduction with ordinary lumping of Markov reward chains, we can eliminate the effect of the speeds and obtain a reduction-like aggregation method for Markov reward chains with silent transitions. Here, we refer to this method as $\tau_{\sim}$-reduction.

One could define a reduction-based method for Markov reward chains with silent transitions by saying that a Markov reward chain with silent transitions $S$ reduces to a Markov reward chain $M$ if all Markov reward chains with fast transitions $\mathrm{F} \in \mathrm{S}$ will $\tau$-reduce to $\mathrm{M}$. However, such is not an efficient reduction method as it is applicable only in the few special cases when all Markov reward chains with fast transitions in a $\sim$-equivalence class $\tau$-reduce to the same Markov reward chain $[10,11]$. For this reason we combine $\tau$-reduction and lumping.

Similarly as for $\tau_{\sim}$-lumping, the result of the $\tau_{\sim}$-reduction should not depend on the representative Markov reward chain with fast transitions. Therefore, a Markov reward chain with silent transitions can be $\tau_{\sim}$-reduced if all Markov 
reward chains with fast transitions in its equivalence class $\tau$-reduce to Markov reward chains that can be ordinary lumped to the same Markov reward chain, as depicted below:

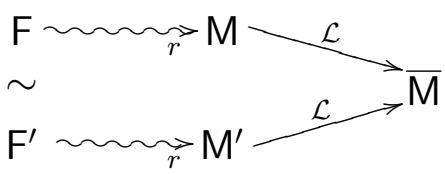

Definition 27 Let S be a Markov reward chain with silent transitions. Let $\mathcal{E}=\left\{E_{1}, \ldots, E_{M}, T\right\}$ be its ergodic partition, and $\mathcal{L}$ a partition of $\left\{E_{1}, \ldots, E_{M}\right\}$. Then $\mathbf{S}$ can be $\tau_{\sim}$-reduced according to $\mathcal{L}$ if and only if there exists a Markov reward chain $\overline{\mathrm{M}}$, such that for every $\mathrm{F} \in \mathrm{S}$, we have that $\mathrm{F} \sim_{r} \mathrm{M} \stackrel{\mathcal{L}}{\rightarrow} \overline{\mathrm{M}}$ for some Markov reward chain $\mathrm{M}$.

In the above situation, we write $\mathrm{S} \underset{\sim}{\sim}{ }_{r} \bar{M}$ and $S \sim_{r} \bar{M}$ if a partition $\mathcal{L}$ exists such that $\mathrm{S} \underset{\sim}{\mathcal{L}_{r}} \overline{\mathrm{M}}$. We note that both $\tau_{\sim}$-lumping and $\tau_{\sim}$-reduction produce the same process when all silent transitions are eliminated, cf. $[10,11]$ for details.

Example 28 The Markov reward chain with fast transitions depicted in Fig. 4a) is $\tau_{\sim}$-reduced to the $\tau_{\sim}$-lumped version of the process from Fig. 6 a). As in the case of $\tau_{\sim}$-lumping above, there is no $\tau_{\sim}$-reduced process of the Markov reward chain with fast transitions in Fig. 4b), as the $\tau$-reduced process depends on the parameters and no further aggregation is possible. To obtain the $\tau_{\sim}$-reduced version of the Markov reward chain with fast transitions depicted in Fig. 4c) we have to assume that $\lambda=\mu$ and $r_{2}=r_{3}$ in order to lump the Markov reward chain from Fig. 7.

\section{Relational Properties}

We investigate the relational properties of the lumping-based aggregation methods. For ordinary lumping, the combination of transitivity and strict confluence ensures that iterative application yields a uniquely determined process. In the case of $\tau$-lumping, in view of Proposition 17, only the limit of the final reduced process is uniquely determined, unless the final process contains no fast transitions. Similarly, for $\tau_{\sim}$-lumping the reduced process is uniquely determined only if it does not contain any silent transitions.

There is no need to investigate the relational properties of the reduction-based methods, since they act in one step (no iteration is possible), in a unique way, between different types of models.

First, we investigate the properties of the relation $\geqslant$ on discontinuous Markov 
reward chains defined by

$$
\mathrm{D}_{1} \geqslant \mathrm{D}_{2} \Longleftrightarrow(\exists \mathcal{L}) \mathrm{D}_{1} \stackrel{\mathcal{L}}{\rightarrow} \mathrm{D}_{2}
$$

The above relation is clearly reflexive, since the trivial partition $\Delta$ is always an ordinary lumping, i.e., $\mathrm{D} \stackrel{\Delta}{\rightarrow} \mathrm{D}$ for any discontinuous Markov reward chain D. Transitivity enables replacement of repeated application of ordinary lumping by a single application using an ordinary lumping that is a composition of the individual lumpings.

Theorem 29 Let $\mathrm{D}$ be a discontinuous Markov reward chain such that $\mathrm{D} \stackrel{\mathcal{L}}{\rightarrow} \overline{\mathrm{D}}$ and $\overline{\mathrm{D}} \stackrel{\overline{\mathcal{L}}}{\rightarrow} \overline{\overline{\mathrm{D}}}$. Then $\mathrm{D} \stackrel{\mathcal{L} \circ \overline{\mathcal{L}}}{\rightarrow} \overline{\overline{\mathrm{D}}}$.

PROOF. Let $\mathrm{D}=(\sigma, \Pi, Q, \rho), \overline{\mathrm{D}}=(\bar{\sigma}, \bar{\Pi}, \bar{Q}, \bar{\rho})$, and $\overline{\overline{\mathrm{D}}}=(\overline{\bar{\sigma}}, \overline{\bar{\Pi}}, \overline{\bar{Q}}, \overline{\bar{\rho}})$. Let $V$ and $\bar{V}$ denote the collector matrices for $\mathcal{L}$ and $\overline{\mathcal{L}}$, respectively. The collector matrix for $\mathcal{L} \circ \overline{\mathcal{L}}$ is $V \bar{V}$. The following lumping conditions hold: $V U \Pi V=\Pi V$, $V U Q V=Q V$ and $V U \rho=\rho$. Also $\bar{\Pi}=U \Pi V, \bar{Q}=U Q V$ and $\bar{\rho}=U \rho$ for any distributor $U$ for $V$. Similarly, it holds that: $\bar{V} \bar{U} \bar{\Pi} \bar{V}=\bar{\Pi} \bar{V}, \bar{V} \bar{U} \bar{Q} \bar{V}=\bar{Q} \bar{V}$ and $\bar{V} \bar{U} \bar{\rho}=\bar{\rho}$. Moreover $\overline{\bar{\Pi}}=\bar{U} \bar{\Pi} \bar{V}, \overline{\bar{Q}}=\bar{U} \bar{Q} \bar{V}$ and $\overline{\bar{\rho}}=\bar{U} \bar{\rho}$ for any distributor $\bar{U}$ for $\bar{V}$.

The iterative application of the ordinary lumping method can be replaced by the ordinary lumping given by the partition $\mathcal{L} \circ \overline{\mathcal{L}}$, that corresponds to the collector matrix $\overline{\bar{V}}=V \bar{V}$. A corresponding distributor is $\overline{\bar{U}}=\bar{U} U$, because $\overline{\bar{U}} \overline{\bar{V}}=\bar{U} U V \bar{V}=I$. That the partition is indeed an ordinary lumping follows from: $\overline{\bar{V}} \overline{\bar{U}} \overline{\bar{V}}=V \bar{V} \bar{U} U \Pi V \bar{V}=V \bar{V} \bar{U} \bar{\Pi} \bar{V}=V \bar{\Pi} \bar{V}=$ $V U \Pi V \bar{V}=\Pi V \bar{V}=\Pi \overline{\bar{V}}$. Similarly, one gets the condition for $Q$, and $\overline{\bar{V}} \overline{\bar{U}} \rho=V \bar{V} \bar{U} U \rho=V \bar{V} \bar{U} \bar{\rho}=V \bar{\rho}=V U \rho=\rho$.

The relation $\geqslant$ on Markov reward chains with fast transitions, defined by

$$
\mathrm{F}_{1} \geqslant \mathrm{~F}_{2} \Longleftrightarrow(\exists \mathcal{L}) \mathrm{F}_{1} \stackrel{\mathcal{L}}{\sim} \mathrm{F}_{2}
$$

is a preorder as well. It is reflexive via the trivial lumping $\Delta$. The following theorem shows the transitivity of the $\tau$-lumping relation.

Theorem 30 Let $\mathrm{F}$ be a Markov reward chain with fast transitions, such that $\mathrm{F} \stackrel{\mathcal{L}}{\sim} \overline{\mathrm{F}}$ and $\overline{\mathrm{F}} \overline{\mathcal{L}} \overline{\overline{\mathrm{F}}}$. Then $\mathrm{F} \stackrel{\mathcal{L} 0 \overline{\mathcal{L}}}{\sim} \overline{\overline{\mathrm{F}}}$.

PROOF. Let $\mathrm{F}=(\sigma, F, S, \rho)$ and $\overline{\mathrm{F}}=(\bar{\sigma}, \bar{F}, \bar{S}, \bar{\rho})$. Denote by $V$ and $\bar{V}$ the collector matrices for $\mathcal{L}$ and $\overline{\mathcal{L}}$, respectively. The collector matrix for $\mathcal{L} \circ \overline{\mathcal{L}}$ is then $\overline{\bar{V}}=V \bar{V}$. Let $W$ and $\bar{W}$ be the corresponding $\tau$-distributors used 
for $\mathrm{F} \stackrel{\mathcal{L}}{\sim} \overline{\mathrm{F}}$ and $\overline{\mathrm{F}} \stackrel{\overline{\mathcal{L}}}{\sim} \overline{\overline{\mathrm{F}}}$, respectively. Since $\tau$-lumping is defined in terms of ordinary lumping, it is sufficient to show that $\overline{\bar{W}}=\bar{W} W$ is a $\tau$-distributor. From Theorem 29 it is a distributor. The condition requiring positive entries corresponding to transient states that lump only with other transient states, can be checked using the explicit description of $\tau$-distributors [11]. It remains to verify the third condition.

Let $\Pi$ and $\bar{\Pi}$ be the ergodic projections of $F$ and $\bar{F}$. Then, $\Pi V W \Pi=\Pi V W$ and $\bar{\Pi} \bar{V} \bar{W} \bar{\Pi}=\bar{\Pi} \bar{V} \bar{W}$. We have that:

$$
\begin{aligned}
\Pi \overline{\bar{V}} \overline{\bar{W}} \Pi & =\Pi V \bar{V} \bar{W} W \Pi=V W \Pi V \bar{V} \bar{W} W \Pi=V \bar{\Pi} \bar{V} \bar{W} W \Pi \\
& =V \bar{\Pi} \bar{V} \bar{W} \bar{\Pi} W \Pi=V \bar{\Pi} \bar{V} \bar{W} W \Pi V W \Pi=V \bar{\Pi} \bar{V} \bar{W} W \Pi V W \\
& =(\text { the same derivation steps backwards) } \\
& =\Pi V \bar{V} \bar{W} W=\Pi \overline{\bar{V}} \bar{W}
\end{aligned}
$$

Similarly, $\tau_{\sim}$-lumping induces a preorder on Markov reward chains with silent transitions defined by

$$
\mathrm{S}_{1} \geqslant \mathrm{~S}_{2} \Longleftrightarrow(\exists \mathcal{L}) \mathrm{S}_{1} \stackrel{\mathcal{L}}{\sim} \mathrm{S}_{2}
$$

Reflexivity again holds due to the trivial partition $\Delta$, while transitivity is a direct consequence of Theorem 30 and the definition of $\tau_{\sim}$-lumping, Definition 19. Thus, we have the following theorem.

Theorem 31 Let $\mathrm{S}$ be a Markov reward chain with silent transitions. Suppose $\mathrm{S} \stackrel{\mathcal{L}}{\sim} \overline{\mathrm{S}}$ and $\overline{\mathrm{S}} \overline{\mathcal{L}} \overline{\overline{\mathrm{S}}}$. Then $\mathrm{S} \stackrel{\mathcal{L} 0 \overline{\mathcal{L}}}{\sim} \overline{\overline{\mathrm{S}}}$.

The lumping preorders also have the strict confluence property. In case of lumping this means that if $\mathrm{P} \stackrel{\mathcal{L}_{1}}{\rightarrow} \mathrm{P}_{1}$ and $\mathrm{P} \stackrel{\mathcal{L}_{2}}{\rightarrow} \mathrm{P}_{2}$, then there exist two partitions $\overline{\mathcal{L}}_{1}$ and $\overline{\mathcal{L}}_{2}$ such that $\mathrm{P}_{1} \stackrel{\mathcal{L}_{1} \circ \overline{\mathcal{L}}_{1}}{\longrightarrow} \overline{\mathrm{P}}$ and $\mathrm{P}_{2} \stackrel{\mathcal{L}_{2} \circ \overline{\mathcal{L}}_{2}}{\longrightarrow} \overline{\mathrm{P}}$. One can prove the strict confluence property by adapting the proof for Markov reward chains, e.g., from [20].

\section{Parallel Composition and Compositionality}

In this section we define parallel composition for each of the models, and prove the compositionality results. The definitions are based on Kronecker products and sums, as for standard Markov reward chains [15,33]. The intuition behind this is that the Kronecker sum represents interleaving, whereas the Kronecker product represents synchronization. Let us first recall the definition of Kronecker product and sum. 
Definition 32 Let $A \in \mathbb{R}^{n_{1} \times n_{2}}$ and $B \in \mathbb{R}^{m_{1} \times m_{2}}$. The Kronecker product of $A$ and $B$ is a matrix $(A \otimes B) \in \mathbb{R}^{n_{1} m_{1} \times n_{2} m_{2}}$ defined by

$$
(A \otimes B)\left[(i-1) m_{1}+k,(j-1) m_{2}+\ell\right]=A[i, j] B[k, \ell]
$$

for $1 \leqslant i \leqslant n_{1}, 1 \leqslant j \leqslant n_{2}, 1 \leqslant k \leqslant m_{1}$ and $1 \leqslant \ell \leqslant m_{2}$.

The Kronecker sum of two square matrices $A \in \mathbb{R}^{n \times n}$ and $B \in \mathbb{R}^{m \times m}$ is a matrix $(A \oplus B) \in \mathbb{R}^{n m \times n m}$ defined by $A \oplus B=A \otimes I^{m}+I^{n} \otimes B$.

Next, we list some basic properties of the Kronecker product and sum.

Proposition 33 ([34]) The following equations hold:

(1) $(A \otimes B)(C \otimes D)=A C \otimes B D$,

(2) $(A+B) \otimes(C+D)=A \otimes C+A \otimes D+B \otimes C+B \otimes D$,

(3) $c(A \otimes B)=(c A \otimes B)=(A \otimes c B)$,

(4) $c(A \oplus B)=(c A \oplus c B)$,

(5) $e^{A \oplus B}=e^{A} \otimes e^{B}$,

(6) $\operatorname{rank}(A \otimes B)=\operatorname{rank}(A) \operatorname{rank}(B)$.

We also need the notion of a Kronecker product of two partitions. Let $\mathcal{L}_{1}$ and $\mathcal{L}_{2}$ be two partitions with corresponding collector matrices $V_{1}$ and $V_{2}$, respectively. Then $\mathcal{L}_{1} \otimes \mathcal{L}_{2}$ denotes the partition corresponding to the collector matrix $V_{1} \otimes V_{2}$.

\subsection{Composing Discontinuous Markov Reward Chains}

First, we present the definition of parallel composition of discontinuous Markov reward chains. The intuition is that 'rates' interleave, and the probabilities of the instantaneous transitions synchronize, i.e., they are independent.

Definition 34 Let $\mathrm{D}_{1}=\left(\sigma_{1}, \Pi_{1}, Q_{1}, \rho_{1}\right)$ and $\mathrm{D}_{2}=\left(\sigma_{2}, \Pi_{2}, Q_{2}, \rho_{2}\right)$ be discontinuous Markov reward chains. Their parallel composition is defined as:

$$
\mathrm{D}_{1} \| \mathrm{D}_{2}=\left(\sigma_{1} \otimes \sigma_{2}, \Pi_{1} \otimes \Pi_{2}, Q_{1} \otimes \Pi_{2}+\Pi_{1} \otimes Q_{2}, \rho_{1} \otimes \mathbf{1}^{\left|\rho_{2}\right|}+\mathbf{1}^{\left|\rho_{1}\right|} \otimes \rho_{2}\right) .
$$

The following theorem shows that the parallel composition of two discontinuous Markov reward chains is well defined.

Theorem 35 Let $\mathrm{D}_{1}$ and $\mathrm{D}_{2}$ be two discontinuous Markov reward chains. Then $\mathrm{D}_{1} \| \mathrm{D}_{2}$ is a discontinuous Markov reward chain. 
PROOF. Let $\mathbf{D}_{1}=\left(\sigma_{1}, \Pi_{1}, Q_{1}, \rho_{1}\right)$ and $\mathbf{D}_{2}=\left(\sigma_{2}, \Pi_{2}, Q_{2}, \rho_{2}\right)$. The initial probability vector $\sigma_{1} \otimes \sigma_{2}$ is a stochastic vector and the reward vector is well defined. Using Proposition 33(1)-(3), it is easy to check that the matrices $\Pi_{1} \otimes \Pi_{2}$ and $Q_{1} \otimes \Pi_{2}+\Pi_{1} \otimes Q_{2}$ satisfy the conditions of Definition 2, i.e., (1) $\left(\Pi_{1} \otimes \Pi_{2}\right) \geq 0$, (2) $\left(\Pi_{1} \otimes \Pi_{2}\right) \cdot \mathbf{1}=\mathbf{1},(3)\left(\Pi_{1} \otimes \Pi_{2}\right)^{2}=\Pi_{1} \otimes \Pi_{2}$, (4) $\left(\Pi_{1} \otimes\right.$ $\left.\Pi_{2}\right) \cdot\left(Q_{1} \otimes \Pi_{2}+\Pi_{1} \otimes Q_{2}\right)=\left(Q_{1} \otimes \Pi_{2}+\Pi_{1} \otimes Q_{2}\right) \cdot\left(\Pi_{1} \otimes \Pi_{2}\right)=Q_{1} \otimes \Pi_{2}+\Pi_{1} \otimes Q_{2}$, (5) $\left(Q_{1} \otimes \Pi_{2}+\Pi_{1} \otimes Q_{2}\right) \cdot \mathbf{1}=\mathbf{0}$, and (6) $Q_{1} \otimes \Pi_{2}+\Pi_{1} \otimes Q_{2}+\left(c_{1}+c_{2}\right) \cdot\left(\Pi_{1} \otimes \Pi_{2}\right)=$ $\left(Q_{1}+c_{1} \Pi_{1}\right) \otimes \Pi_{2}+\Pi_{1} \otimes\left(Q_{2}+c_{2} \Pi_{2}\right) \geq 0$ for $c_{1}, c_{2} \geq 0$ such that $Q_{1}+c_{1} \Pi_{1} \geq 0$ and $Q_{2}+c_{2} \Pi_{2} \geq 0$.

In the special case, when both discontinuous Markov reward chains are continuous, their parallel composition is again a Markov reward chain as defined in [15]. Moreover, the following property shows that the parallel composition of two discontinuous Markov reward chains has a transition matrix that is the Kronecker product of the individual transition matrices, corresponding to the intuition that the Kronecker product represents synchronization. This justifies the definition of the parallel composition.

Theorem 36 Let $\mathrm{D}_{1}$ and $\mathrm{D}_{2}$ be two discontinuous Markov reward chains with transition matrices $P_{1}(t)$ and $P_{2}(t)$, respectively. Then the transition matrix of $\mathrm{D}_{1} \| \mathrm{D}_{2}$ is given by $P_{1}(t) \otimes P_{2}(t)$.

PROOF. Let $\mathrm{D}_{1}=\left(\sigma_{1}, \Pi_{1}, Q_{1}, \rho_{1}\right)$ and $\mathrm{D}_{2}=\left(\sigma_{2}, \Pi_{2}, Q_{2}, \rho_{2}\right)$. As the matrices $Q_{1} \otimes \Pi_{2}$ and $\Pi_{1} \otimes Q_{2}$ commute, and $P_{i}(t) \Pi_{i}=\Pi_{i} P_{i}(t)=P_{i}(t)$, we derive:

$$
\begin{aligned}
& \left(\Pi_{1} \otimes \Pi_{2}\right) e^{\left(Q_{1} \otimes \Pi_{2}+\Pi_{1} \otimes Q_{2}\right) t} \\
= & \left(\Pi_{1} \otimes \Pi_{2}\right)\left(e^{\left(Q_{1} \otimes \Pi_{2}\right) t} e^{\left(\Pi_{1} \otimes Q_{2}\right) t}\right) \\
= & \left(\Pi_{1} \otimes \Pi_{2}\right)\left(\sum_{n=0}^{\infty}\left(Q_{1} \otimes \Pi_{2}\right)^{n} t^{n} / n !\right)\left(\sum_{n=0}^{\infty}\left(\Pi_{1} \otimes Q_{2}\right)^{n} t^{n} / n !\right) \\
= & \left(\Pi_{1} \otimes \Pi_{2}\right)\left(I \otimes I+\sum_{n=1}^{\infty}\left(Q_{1} \otimes \Pi_{2}\right)^{n} t^{n} / n !\right)\left(I \otimes I+\sum_{n=1}^{\infty}\left(\Pi_{1} \otimes Q_{2}\right)^{n} t^{n} / n !\right) \\
= & \left(\Pi_{1} \otimes \Pi_{2}\right)\left(I \otimes I+\sum_{n=1}^{\infty}\left(Q_{1}^{n} \otimes \Pi_{2}^{n}\right) t^{n} / n !\right)\left(I \otimes I+\sum_{n=1}^{\infty}\left(\Pi_{1}^{n} \otimes Q_{2}^{n}\right) t^{n} / n !\right) \\
= & \left(\Pi_{1} \otimes \Pi_{2}\right)\left(I \otimes I+\sum_{n=1}^{\infty}\left(Q_{1}^{n} \otimes \Pi_{2}\right) t^{n} / n !\right)\left(I \otimes I+\sum_{n=1}^{\infty}\left(\Pi_{1} \otimes Q_{2}^{n}\right) t^{n} / n !\right) \\
= & \left(\Pi_{1} \otimes \Pi_{2}\right)\left(I \otimes I+\left(\sum_{n=1}^{\infty} Q_{1}^{n} t^{n} / n !\right) \otimes \Pi_{2}\right)\left(I \otimes I+\Pi_{1} \otimes \sum_{n=1}^{\infty} Q_{2}^{n} t^{n} / n !\right) \\
= & \left(\Pi_{1} \otimes \Pi_{2}\right)\left(I \otimes I+\left(e_{1} \bar{t}-I\right) \otimes \Pi_{2}\right)\left(I \otimes I+\Pi_{1} \otimes\left(e^{Q_{2} t}-I\right)\right) \\
= & \left(\Pi_{1} \otimes \Pi_{2}\right)\left(I \otimes I+e^{Q_{1} t} \otimes \Pi_{2}-I \otimes \Pi_{2}\right)\left(I \otimes I+\Pi_{1} \otimes e^{Q_{2} t}-\Pi_{1} \otimes I\right) \\
= & \left(\Pi_{1} \otimes \Pi_{2}+P_{1}(t) \otimes \Pi_{2}-\Pi_{1} \otimes \Pi_{2}\right)\left(I \otimes I+\Pi_{1} \otimes e^{Q_{2} t}-\Pi_{1} \otimes I\right) \\
= & \left(P_{1}(t) \otimes \Pi_{2}\right)\left(I \otimes I+\Pi_{1} \otimes e_{2} t-\Pi_{1} \otimes I\right) \\
= & \left(P_{1}(t) \otimes \Pi_{2}+P_{1}(t) \otimes P_{2}(t)-P_{1}(t) \otimes \Pi_{2}\right) \\
= & P_{1}(t) \otimes P_{2}(t) .
\end{aligned}
$$

Remark 37 We can motivate Definition 34 also from another perspective. By the standard probabilistic (i.e., non-matrix) representation of discontinuous Markov reward chain the same notion can be obtained by the following analysis. Let $\{X(t) \mid t \geqslant 0\}$ and $\{Y(t) \mid t \geqslant 0\}$ be two discontinuous Markov reward chains defined on state spaces $S_{X}$ and $S_{Y}$, respectively. Their parallel composition can be defined as the stochastic process $\{(X \| Y)(t) \mid t \geqslant 0\}$ 
with the state space $S_{X} \times S_{Y}$, such that $(X \| Y)(t)=(x, y)$ if and only if $X(t)=x$ and $Y(t)=y$. One can show that this process is again a discontinuous Markov reward chain with transition matrix equal to the Kronecker product of the transition matrices of $\{X(t) \mid t \geqslant 0\}$ and $\{Y(t) \mid t \geqslant 0\}$. It is known that the matrices $\Pi$ and $Q$ characterizing a transition matrix $P(t)$ are obtained as $\Pi=\lim _{t \rightarrow 0} P(t)$ and $Q=\lim _{h \rightarrow 0}(P(h)-\Pi) / h$ [13]. Applying this result on the transition matrix of $\{(X \| Y)(t) \mid t \geqslant 0\}$ and using the definition of $(X \| Y)(0)$ we obtain the first three components of the quadruple from Definition 34. The reward vector for the parallel composition encodes the assumption that the reward rate in $(x, y)$ is the sum of the reward rates in $x$ and $y$.

It is easy to see that the expected reward of the parallel composition is the sum of the expected rewards of the components. Using Proposition 33(1) and (2) we have $\left(\sigma_{1} \otimes \sigma_{2}\right)\left(P_{1}(t) \otimes P_{2}(t)\right)\left(\rho_{1} \otimes \mathbf{1}+\mathbf{1} \otimes \rho_{2}\right)=\sigma_{1} P_{1}(t) \rho_{1} \otimes \sigma_{1} P_{1}(t) \mathbf{1}+$ $\sigma_{2} P_{2}(t) \mathbf{1} \otimes \sigma_{2} P_{2}(t) \rho_{2}=R_{1}(t) \otimes 1+1 \otimes R_{2}(t)=R_{1}(t)+R_{2}(t)$.

The following theorem shows that both lumping and reduction are compositional with respect to the parallel composition of discontinuous Markov reward chains.

Theorem 38 If $\mathrm{D}_{1} \stackrel{\mathcal{L}_{1}}{\rightarrow} \overline{\mathrm{D}}_{1}$ and $\mathrm{D}_{2} \stackrel{\mathcal{L}_{2}}{\rightarrow} \overline{\mathrm{D}}_{2}$, then $\mathrm{D}_{1}\left\|\mathrm{D}_{2} \stackrel{\mathcal{L}_{1} \otimes \mathcal{L}_{2}}{\rightarrow} \overline{\mathrm{D}}_{1}\right\| \overline{\mathrm{D}}_{2}$. Also, if $\mathrm{D}_{1} \rightarrow_{r} \mathrm{M}_{1}$ and $\mathrm{D}_{2} \rightarrow_{r} \mathrm{M}_{2}$, then $\mathrm{D}_{1}\left\|\mathrm{D}_{2} \rightarrow_{r} \mathrm{M}_{1}\right\| \mathrm{M}_{2}$.

PROOF. Let $\mathrm{D}_{1}=\left(\sigma_{1}, \Pi_{1}, Q_{1}, \rho_{1}\right), \quad \overline{\mathrm{D}}_{1}=\left(\bar{\sigma}_{1}, \bar{\Pi}_{1}, \bar{Q}_{1}, \bar{\rho}_{1}\right), \quad \mathrm{D}_{2}=$ $\left(\sigma_{2}, \Pi_{2}, Q_{2}, \rho_{2}\right)$, and $\overline{\mathrm{D}}_{2}=\left(\bar{\sigma}_{2}, \bar{\Pi}_{2}, \bar{Q}_{2}, \bar{\rho}_{2}\right)$. We first prove the compositionality of lumping. We show that $\mathcal{L}_{1} \otimes \mathcal{L}_{2}$ is an ordinary lumping of

$$
\mathrm{D}_{1} \| \mathrm{D}_{2}=\left(\sigma_{1} \otimes \sigma_{2}, \Pi_{1} \otimes \Pi_{2}, Q_{1} \otimes \Pi_{2}+\Pi_{1} \otimes Q_{2}, \rho_{1} \otimes \mathbf{1}+\mathbf{1} \otimes \rho_{2}\right) .
$$

Let $U_{1}, U_{2}$, and $U_{1} \otimes U_{2}$ be distributors and $V_{1}, V_{2}$, and $V_{1} \otimes V_{2}$ be the collectors for $\mathcal{L}_{1}, \mathcal{L}_{2}$, and $\mathcal{L}_{1} \otimes \mathcal{L}_{2}$, respectively. By using the lumping conditions and Proposition 33(1) and (2) we have that

$$
\begin{aligned}
& \left(V_{1} \otimes V_{2}\right)\left(U_{1} \otimes U_{2}\right)\left(\Pi_{1} \otimes \Pi_{2}\right)\left(V_{1} \otimes V_{2}\right) \\
& =\left(V_{1} U_{1} \Pi_{1} V_{1} \otimes V_{2} U_{2} \Pi_{2} V_{2}\right) \\
& =\left(\Pi_{1} V_{1} \otimes \Pi_{2} V_{2}\right)=\left(\Pi_{1} \otimes \Pi_{2}\right)\left(V_{1} \otimes V_{2}\right) \\
& \left(V_{1} \otimes V_{2}\right)\left(U_{1} \otimes U_{2}\right)\left(Q_{1} \otimes \Pi_{2}+\Pi_{1} \otimes Q_{2}\right)\left(V_{1} \otimes V_{2}\right) \\
& \quad=V_{1} U_{1} Q_{1} V_{1} \otimes V_{2} U_{2} \Pi_{2} V_{2}+V_{1} U_{1} \Pi_{1} V_{1} \otimes V_{2} U_{2} Q_{2} V_{2} \\
& =Q_{1} V_{1} \otimes \Pi_{2} V_{2}+\Pi_{1} V_{1} \otimes Q_{2} V_{2} \\
& =\left(Q_{1} \otimes \Pi_{2}+\Pi_{1} \otimes Q_{2}\right)\left(V_{1} \otimes V_{2}\right)
\end{aligned}
$$




$$
\begin{aligned}
\left(V_{1} \otimes\right. & \left.V_{2}\right)\left(U_{1} \otimes U_{2}\right)\left(\rho_{1} \otimes \mathbf{1}+\mathbf{1} \otimes \rho_{2}\right) \\
& =\left(V_{1} U_{1} \rho_{1} \otimes V_{2} U_{2} \mathbf{1}+V_{1} U_{1} \mathbf{1} \otimes V_{2} U_{2} \rho_{2}\right) \\
& =\rho_{1} \otimes \mathbf{1}+\mathbf{1} \otimes \rho_{2} .
\end{aligned}
$$

Next, we prove that the lumped parallel composition is the parallel composition of the lumped components. We have, by Proposition 33(1) and (2),

$$
\begin{aligned}
& \left(U_{1} \otimes U_{2}\right)\left(\Pi_{1} \otimes \Pi_{2}\right)\left(V_{1} \otimes V_{2}\right)=\bar{\Pi}_{1} \otimes \bar{\Pi}_{2} \text { and } \\
& \left(U_{1} \otimes U_{2}\right)\left(Q_{1} \otimes \Pi_{2}+\Pi_{1} \otimes Q_{2}\right)\left(V_{1} \otimes V_{2}\right)=\bar{Q}_{1} \otimes \bar{\Pi}_{2}+\bar{\Pi}_{1} \otimes \bar{Q}_{2} .
\end{aligned}
$$

Next, we consider reduction. Let $\Pi_{1}=R_{1} L_{1}$ and $\Pi_{2}=R_{2} L_{2}$ be some canonical product decompositions. Put $L=L_{1} \otimes L_{2}$ and $R=R_{1} \otimes R_{2}$. Note that $L \geq 0$ and $R \geq 0$ because $L_{1}, L_{2}, R_{1}, R_{2} \geq 0$. We also have $L \cdot \mathbf{1}=\left(L_{1} \otimes L_{2}\right) \cdot(\mathbf{1} \otimes \mathbf{1})=$ $L_{1} \cdot \mathbf{1} \otimes L_{2} \cdot \mathbf{1}=\mathbf{1} \otimes \mathbf{1}=\mathbf{1}$. Since $\operatorname{rank}(A \otimes B)=\operatorname{rank}(A) \cdot \operatorname{rank}(B)$ by Proposition 33(6), we get that $(L, R)$ is a canonical product decomposition of $\Pi=\Pi_{1} \otimes \Pi_{2}$. Reducing $D_{1} \| D_{2}$ using the canonical product decomposition $(L, R)$ gives us $\mathrm{M}_{1} \| \mathrm{M}_{2}$.

\subsection{Composing Markov Reward Chains with Fast Transitions}

We now present the definition of the parallel composition of Markov reward chains with fast transitions. It comprises Kronecker sums of the generator matrices, i.e., interleaving of the rates for both slow and fast transitions.

Definition 39 Let $\boldsymbol{F}_{1}=\left(\sigma_{1}, S_{1}, F_{1}, \rho_{1}\right)$ and $\mathbf{F}_{2}=\left(\sigma_{2}, S_{2}, F_{2}, \rho_{2}\right)$ be two Markov reward chains with fast transitions. Then their parallel composition is defined as

$$
\mathrm{F}_{1} \| \mathrm{F}_{2}=\left(\sigma_{1} \otimes \sigma_{2}, S_{1} \oplus S_{2}, F_{1} \oplus F_{2}, \rho_{1} \otimes \mathbf{1}+\mathbf{1} \otimes \rho_{2}\right)
$$

It is not difficult to see that the parallel composition of Markov reward chains with fast transitions is well-defined.

Example 40 In Fig. 8 we present an example of parallel composition of two Markov reward chains with fast transitions: $8 c$ ) is the parallel composition of 8a) and 8b), the same Markov reward chains with fast transitions from the example in Fig. 4. For readability the rewards of 8c) are omitted. They are given by the vector

$$
\left(r_{1}+r_{4}, r_{1}+r_{5}, r_{1}, r_{2}+r_{4}, r_{2}+r_{5}, r_{2}, r_{3}+r_{4}, r_{3}+r_{5}, r_{3}\right)
$$



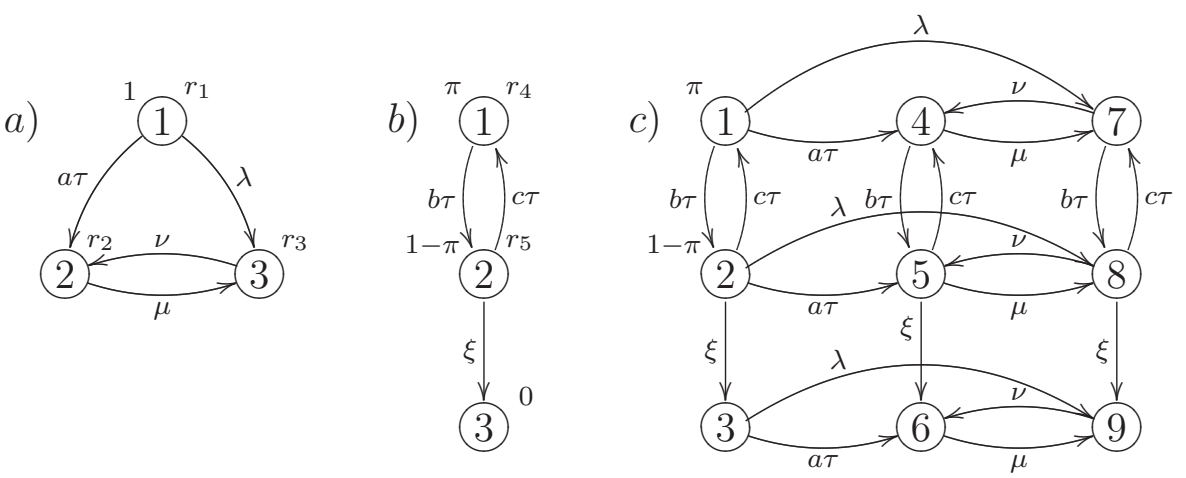

Fig. 8. Parallel composition of Markov reward chains with fast transitions

Having defined parallel composition for both models, we show how they are related: the limit of the parallel composition of two Markov reward chains with fast transitions is the parallel composition of the limits of the components (that are discontinuous Markov reward chains). Hence, a continuity property of the parallel composition holds as stated in the next result.

Theorem 41 Let $F_{1} \rightarrow_{\infty} D_{1}$ and $F_{2} \rightarrow_{\infty} D_{2}$. Then $F_{1}\left\|F_{2} \rightarrow_{\infty} D_{1}\right\| D_{2}$.

PROOF. Let $\mathrm{F}_{1}=\left(\sigma_{1}, S_{1}, F_{1}, \rho_{1}\right)$ and $\mathrm{F}_{2}=\left(\sigma_{2}, S_{2}, F_{2}, \rho_{2}\right)$, and let their corresponding limits be $\mathrm{D}_{1}=\left(\sigma_{1}, \Pi_{1}, Q_{1}, \Pi_{1} \rho_{1}\right)$ and $\mathrm{D}_{2}=\left(\sigma_{2}, \Pi_{2}, Q_{2}, \Pi_{2} \rho_{2}\right)$. Using Proposition 33(4) and (5) we get that $\Pi_{1} \otimes \Pi_{2}$ is the ergodic projection of $F_{1} \oplus F_{2}$, i.e. $\lim _{t \rightarrow \infty} e^{\left(F_{1} \oplus F_{2}\right) t}=\Pi_{1} \otimes \Pi_{2}$. As before, using the distributivity of the Kronecker product and the fact that $\Pi_{1}$ is a stochastic matrix, we derive $Q_{1} \otimes \Pi_{2}+\Pi_{2} \otimes Q_{1}=\left(\Pi_{1} \otimes \Pi_{2}\right)\left(S_{1} \oplus S_{2}\right)\left(\Pi_{1} \otimes \Pi_{2}\right)$ and $\left(\Pi_{1} \otimes \Pi_{2}\right)\left(\rho_{1} \otimes \mathbf{1}+\mathbf{1} \otimes \rho_{2}\right)=$ $\Pi_{1} \rho_{1} \otimes \mathbf{1}+\mathbf{1} \otimes \Pi_{2} \rho_{2}$.

Next we show compositionality of $\tau$-lumping and $\tau$-reduction with respect to the parallel composition of Markov reward chains with fast transitions.

Theorem 42 If $\mathrm{F}_{1} \stackrel{\mathcal{L}_{1}}{\rightarrow} \bar{F}_{1}$ and $\mathrm{F}_{2} \stackrel{\mathcal{L}_{2}}{\rightarrow} \bar{F}_{2}$, then $\mathrm{F}_{1}\left\|\mathrm{~F}_{2} \stackrel{\mathcal{L}_{1} \otimes \mathcal{L}_{2}}{\longrightarrow} \overline{\mathrm{F}}_{1}\right\| \bar{F}_{2}$. Also, if $\mathrm{F}_{1} \sim_{r} \mathrm{M}_{1}$ and $\mathrm{F}_{2} \sim_{r} \mathrm{M}_{2}$, then $\mathrm{F}_{1}\left\|\mathrm{~F}_{2} \sim_{r} \mathrm{M}_{1}\right\| \mathrm{M}_{2}$.

PROOF. Let $\mathrm{F}_{1}=\left(\sigma_{1}, S_{1}, F_{1}, \rho_{1}\right), \mathrm{F}_{2}=\left(\sigma_{2}, S_{2}, F_{2}, \rho_{2}\right), \overline{\mathbf{F}}_{1}=\left(\bar{\sigma}_{1}, \bar{S}_{1}, \bar{F}_{1}, \bar{\rho}_{1}\right)$, and $\overline{\mathrm{F}}_{2}=\left(\bar{\sigma}_{2}, \bar{S}_{2}, \bar{F}_{2}, \bar{\rho}_{2}\right)$. By Theorem 38 and the continuity result Theorem 41 , we get that $\mathcal{L}_{1} \otimes \mathcal{L}_{2}$ is a $\tau$-lumping for $\mathrm{F}_{1} \| \mathrm{F}_{2}$. Let $W_{1}$ and $W_{2}$ be the $\tau$-distributors used for the $\tau$-lumped processes in the assumption, respectively. By Definition 14, Theorem 41, and Definition 34 for the parallel composition of discontinuous Markov reward chains, we have that $W_{1} \otimes W_{2}$ is a $\tau$-distributor for $\mathrm{F}_{1} \| \mathrm{F}_{2}$. The $\tau$-lumped process corresponding to $W_{1} \otimes W_{2}$ is exactly $\overline{\mathrm{F}}_{1} \| \overline{\mathrm{F}}_{2}$. 
We next show the compositionality of $\tau$-reduction. Let $\Pi_{1}=R_{1} L_{1}$ and $\Pi_{2}=R_{2} L_{2}$ be the canonical product decompositions of $\Pi_{1}=\lim _{t \rightarrow \infty} e^{F_{1} t}$ and $\Pi_{2}=\lim _{t \rightarrow \infty} e^{F_{2} t}$, respectively. Put $L=L_{1} \otimes L_{2}$ and $R=R_{1} \otimes R_{2}$. Then $(L, R)$ is a canonical product decomposition of $\Pi=\Pi_{1} \otimes \Pi_{2}$, as in the proof of Theorem 38. This canonical product decomposition applied to $F_{1} \| F_{2}$ produces $\mathrm{M}_{1} \| \mathrm{M}_{2}$ as the $\tau$-reduced process.
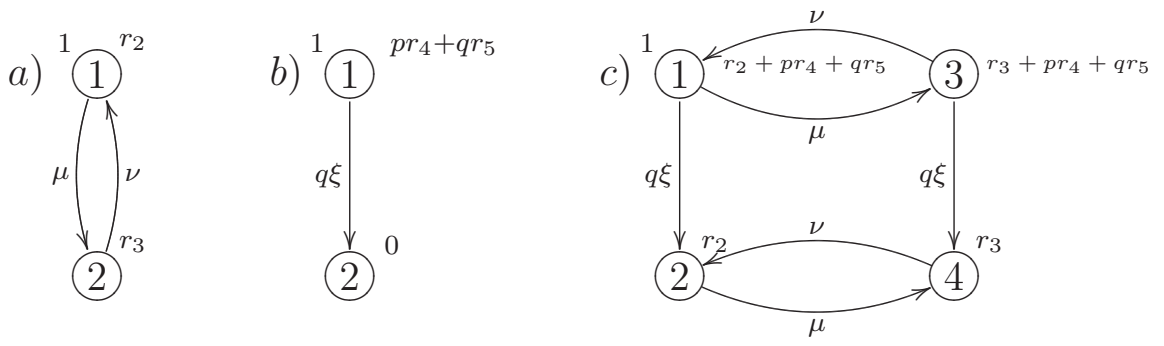

Fig. 9. Aggregated Markov reward chains with fast transitions

Example 43 In Fig. 9 we present the aggregated versions of the Markov reward chains with fast transitions from Fig. 8. As expected by Theorem 42, the Markov reward chain with fast transitions in $9 c$ ) is the parallel composition of the Markov reward chains with fast transitions in 9a) and 9b) with $p=\frac{c}{b+c}$ and $q=\frac{b}{b+c}$. The aggregated versions can be obtained by either applying $\tau$ reduction or $\tau$-lumping as already discussed in Examples 18 and 26. See [10,11] for more details on the relationship between lumping-based and reduction-based aggregation methods. The $\tau$-lumpings used are $\{\{1,2\},\{3\}\}$ for 8a) and 8b), and $\{\{1,2,4,5\},\{3,6\},\{7,8\},\{9\}\}$ for $8 c)$.

\subsection{Composing Markov Reward Chains with Silent Transitions}

We define the parallel composition of two Markov reward chains with silent transitions via the equivalence class of the parallel composition of the representative Markov reward chains with fast transitions.

Definition 44 Let $\mathrm{S}_{1}=\left(\sigma_{1}, S_{1}, \mathcal{F}_{1}, \rho_{1}\right)$ and $\mathrm{S}_{2}=\left(\sigma_{2}, S_{2}, \mathcal{F}_{2}, \rho_{2}\right)$ be two Markov reward chains with silent transitions. Then their parallel composition is defined as

$$
\mathrm{S}_{1} \| \mathrm{S}_{2}=\left(\sigma_{1} \otimes \sigma_{2}, S_{1} \oplus S_{2}, \mathcal{F}_{1} \oplus \mathcal{F}_{2}, \rho_{1} \otimes \mathbf{1}+\mathbf{1} \otimes \rho_{2}\right),
$$

where $\mathcal{F}_{1} \oplus \mathcal{F}_{2}$ denotes the equivalence class of $F_{1} \oplus F_{2}$ with respect to $\sim$, for some $F_{1} \in \mathcal{F}_{1}$ and $F_{2} \in \mathcal{F}_{2}$.

The parallel composition of Markov reward chains with silent transitions is well defined as the Kronecker sum respects the equivalence $\sim$. Next we state 
the compositionality result for $\tau_{\sim}$-lumping and $\tau_{\sim}$-reduction. It is a direct consequence of Theorem 42 for compositionality of $\tau$-lumping and $\tau$-reduction, and compositionality of ordinary lumping for standard Markov reward chain as a special case of Theorem 38 .

Theorem 45 Let $\mathrm{S}_{1}$ and $\mathrm{S}_{2}$ be two Markov reward chains with silent transitions. If $\mathrm{S}_{1} \stackrel{\mathcal{L}_{1}}{\rightarrow} \overline{\mathrm{S}}_{1}$ and $\mathrm{S}_{2} \stackrel{\mathcal{L}_{2}}{\rightarrow} \overline{\mathrm{S}}_{2}$, then $\mathrm{S}_{1}\left\|\mathrm{~S}_{2} \stackrel{\mathcal{L}_{1} \otimes \mathcal{L}_{2}}{\rightarrow} \overline{\mathrm{S}}_{1}\right\| \overline{\mathrm{S}}_{2}$. Also, if $\mathrm{S}_{1} \stackrel{\mathcal{L}_{1}}{\rightarrow} \mathrm{M}_{1}$ and $\mathrm{S}_{2} \stackrel{\mathcal{L}_{2}}{\sim}{ }_{r} \mathrm{M}_{2}$, then $\mathrm{S}_{1}\left\|\mathrm{~S}_{2} \stackrel{\mathcal{L}_{1} \otimes \mathcal{L}_{2}}{\rightarrow}{ }_{r} \mathrm{M}_{1}\right\| \mathrm{M}_{2}$.

\section{Conclusion}

We considered three types of performance models. Markov reward chains with fast transitions are our central model used for analyzing systems with stochastic and instantaneous probabilistic transitions. Their limits are the discontinuous Markov reward chains. Their quotients are the Markov reward chains with silent transitions which can be used for the analysis of systems with stochastic transitions and non-deterministic (internal) $\tau$ steps.

For each type of models, we presented two aggregation methods: lumping and reduction for discontinuous Markov reward chains, $\tau$-lumping and $\tau$ reduction for Markov reward chains with fast transitions, and $\tau_{\sim}$-lumping and $\tau_{\sim}$-reduction for Markov reward chains with silent transitions. In short, the contributions of the paper are the following.

- A definition of parallel composition of discontinuous Markov reward chains, Markov reward chains with fast transitions, and Markov reward chains with silent transitions allowing for compositional modeling.

- Identification of preorder properties of the aggregation methods for all types of models.

- Compositionality theorems for each type of models and each corresponding aggregation preorder, and a continuity property of the parallel compositions.

The results on compositionality are summarized in Fig. 10 which is justified by the Theorems 29-45, as well as by Proposition 17 and Proposition 25.

Further work focuses on the analysis of models that combine stochastic transitions and (non-internal) action labeled transitions, so that in addition to interleaving, synchronization can be expressed too. 


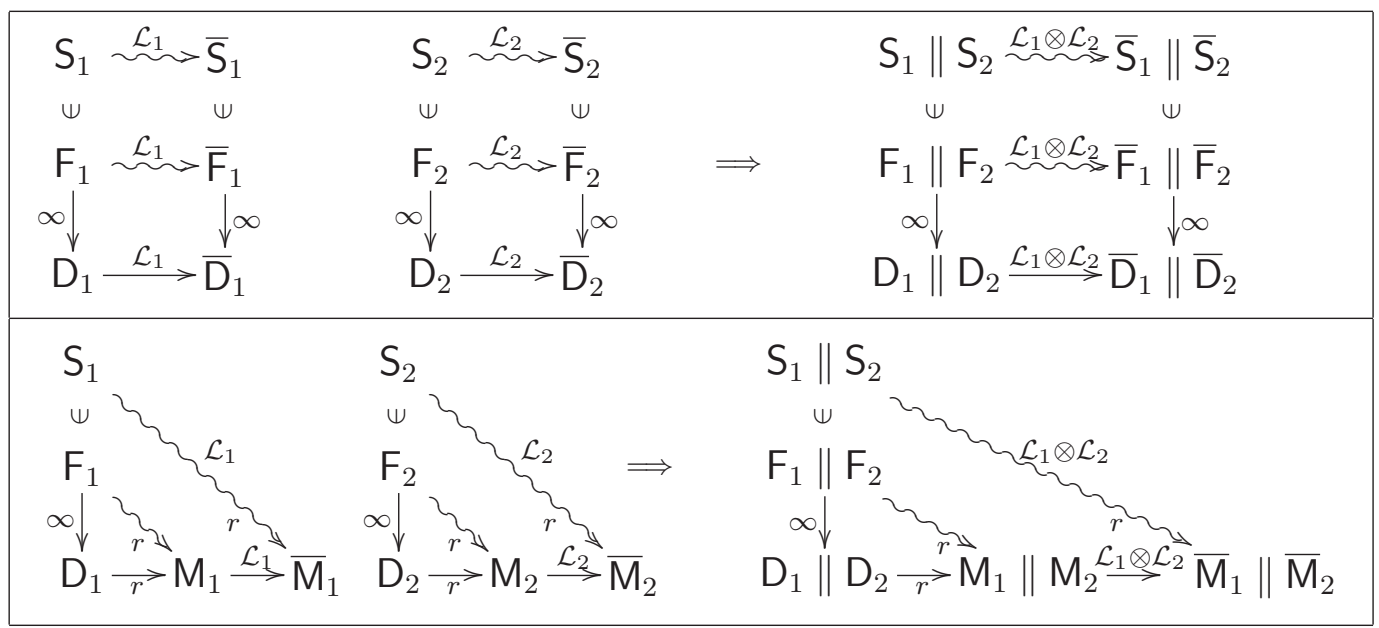

Fig. 10. Summary compositionality results

\section{References}

[1] R. A. Howard, Semi-Markov and Decision Processes, Wiley, 1971.

[2] H. Hermanns, Interactive Markov Chains: The Quest for Quantified Quality, Vol. 2428 of Lecture Notes in Computer Science, Springer, 2002.

[3] J. Hillston, A Compositional Approach to Performance Modelling, Cambridge University Press, 1996.

[4] M. Ajmone Marsan, G. Balbo, G. Conte, S. Donatelli, G. Franceschinis, Modelling with Generalized Stochastic Petri Nets, Wiley, 1995.

[5] G. Ciardo, J. Muppala, K. S. Trivedi, On the solution of GSPN reward models, Performance Evaluation 12 (1991) 237-253.

[6] S.-H. Wu, S. A. Smolka, E. Stark, Composition and behaviors of probabilistic I/O automata, Theoretical Computer Science 176 (1997) 1-38.

[7] L. Cheung, N. Lynch, R. Segala, F. Vaandrager, Switched PIOA: Parallel composition via distributed scheduling, Theoretical Compututer Science 365 (2006) 83-108.

[8] B. Plateau, K. Atif, Stochastic automata network of modeling parallel systems, IEEE Transactions on Software Engineering 17 (1991) 1093-1108.

[9] J. Markovski, N. Trčka, Lumping Markov chains with silent steps, in: Proceedings of QEST'06, IEEE Computer Society, Riverside, 2006, pp. 221230.

[10] J. Markovski, N. Trčka, Aggregation methods for Markov reward chains with fast and silent transitions, in: F. Bause, P. Buchholz (Eds.), Proceedings of MMB 2008, VDE Verlag, 2008.

[11] N. Trčka, Silent steps in transition systems and Markov chains, Ph.D. thesis, Eindhoven University of Technology (2007). 
[12] W. Doeblin, Sur l'équation matricielle $A(t+s)=A(t) \cdot A(s)$ et ses applications aux probabilités en chaine, Bulletin des Sciences Mathématiques 62 (1938) 2132.

[13] M. Coderch, A. S. Willsky, S. S. Sastry, D. A. Castanon, Hierarchical aggregation of singularly perturbed finite state Markov processes, Stochastics 8 (1983) 259-289.

[14] H. H. Ammar, Y. F. Huang, R. W. Liu, Hierarchical models for systems reliability, maintainability, and availability, IEEE Transactions on Circuits and Systems 34 (1987) 629-638.

[15] P. Buchholz, Markovian process algebra: composition and equivalence, in: Proceedings of PAPM 94, Universität Erlangen-Nürnberg, 1994, pp. 11-30.

[16] P. Buchholz, Structured analysis techniques for large Markov chains, in: Proceedings of SMCTools 2006, Vol. 201 of ACM International Conference Proceedings Series, Pisa, 2006, pp. 2-10.

[17] W. J. Stewart, Introduction to the numerical solution of Markov chains, Princeton University Press, 1994.

[18] J. G. Kemeny, J. L. Snell, Finite Markov chains, Springer, 1976.

[19] P. Buchholz, Exact and ordinary lumpability in finite Markov chains, Journal of Applied Probability 31 (1994) 59-75.

[20] A. Sokolova, E. P. de Vink, On relational properties of lumpability, in: Proceedings of 4th PROGRESS symposium on Embedded Systems, Utrecht, The Netherlands, 2003.

[21] F. Delebecque, J. P. Quadrat, Optimal control of Markov chains admitting strong and weak interactions, Automatica 17 (1981) 281-296.

[22] F. Delebecque, A reduction process for perturbed Markov chains, SIAM Journal of Applied Mathematics 2 (1983) 325-330.

[23] J. Markovski, A. Sokolova, N. Trcka, E. de Vink, Compositionality for Markov reward chains with fast transitions, in: K. Wolter (Ed.), Proceedings of EPEW 2007, Vol. 4748 of Lecture Notes of Computer Science, Springer, 2007, pp. 1832 .

[24] C. Baier, B. Haverkort, H. Hermanns, J.-P. Katoen, Model checking meets performance evaluation, SIGMETRICS Performance Evaluation Review 32 (2005) 10-15.

[25] M. Kwiatkowska, G. Norman, D. Parker, PRISM: Probabilistic symbolic model checker, in: Proceedings TOOLS 2002, Springer, 2002, pp. 200-204.

[26] H. Hermanns, J.-P. Katoen, J. Meyer-Kayser, M. Siegle, A tool for model checking Markov chains, Software Tools for Technology Transfer 4 (2003) 153172 . 
[27] J. L. Doob, Stochastic Processes, Wiley, 1953.

[28] K. L. Chung, Markov Chains with Stationary Probabilities, Springer, 1967.

[29] E. Hille, R. S. Phillips, Functional Analysis and Semi-Groups, AMS, 1957.

[30] R. Milner, A Calculus of Communicating Systems, Springer, 1982.

[31] J. Baeten, W. Weijland, Process Algebra, No. 18 in Cambridge Tracts in Theoretical Computer Science, Cambridge University Press, 1990.

[32] V. Nicola, Lumping in Markov reward processes, IBM Research Report RC 14719, IBM (1989).

[33] P. Buchholz, P. Kemper, Kronecker based matrix representations for large Markov chains, in: Validation of Stochastic Systems, Vol. 2925 of Lecture Notes in Computer Science, Springer, 2004, pp. 256-295.

[34] A. Graham, Kronecker Products and Matrix Calculus With Applications, Ellis Horwood, 1981. 